\title{
Coherent elastic neutrino-nucleus scattering at the European Spallation Source
}

\author{
D. Baxter, ${ }^{a}$ J.I. Collar, ${ }^{a}$ P. Coloma, ${ }^{b}$ C.E. Dahl, ${ }^{c, d}$ I. Esteban, ${ }^{e}$ P. Ferrario,,${ }^{f, g}$
}

\section{J.J. Gomez-Cadenas, ${ }^{f, g}$ M.C. Gonzalez-Garcia, ${ }^{e, h, i, j}$ A.R.L. Kavner, ${ }^{a}$ C.M. Lewis, ${ }^{a}$} F. Monrabal, ${ }^{f, g}$ J. Muñoz Vidal, ${ }^{f}$ P. Privitera, ${ }^{a}$ K. Ramanathan ${ }^{a}$ and J. Renner ${ }^{k}$

${ }^{a}$ Enrico Fermi Institute, Kavli Institute for Cosmological Physics, and Department of Physics, University of Chicago, Chicago, Illinois 60637, U.S.A.

${ }^{b}$ Instituto de Física Corpuscular, Universitat de Valéncia and CSIC, Edificio Institutos Investigación, Catedrático José Beltrán 2, 46980 Valencia, Spain

${ }^{c}$ Department of Physics and Astronomy, Northwestern University,

Evanston, Illinois 60208, U.S.A.

${ }^{d}$ Fermi National Accelerator Laboratory,

Batavia, Illinois 60510, U.S.A.

${ }^{e}$ Departament de Fisica Quantica i Astrofisica and Institut de Ciencies del Cosmos, Universitat de Barcelona, Diagonal 647, E-08028 Barcelona, Spain

${ }^{f}$ Donostia International Physics Center (DIPC),

Paseo Manuel Lardizabal, 4, Donostia-San Sebastián, E-20018, Spain

${ }^{g}$ Ikerbasque, Basque Foundation for Science,

Bilbao, E-48013, Spain

${ }^{h}$ Institució Catalana de Recerca $i$ Estudis Avancats (ICREA),

Pg. Lluis Companys 23, 08010 Barcelona, Spain

${ }^{i}$ C.N. Yang Institute for Theoretical Physics, Stony Brook University, Stony Brook NY11794-3849, U.S.A.

${ }^{j}$ Donostia International Physics Center (DIPC),

Paseo Manuel Lardizabal, 4, Donostia-San Sebastián, E-20018, Spain

${ }^{k}$ Instituto Gallego de Física de Altas Energías, Univ. de Santiago de Compostela, Campus sur, Rúa Xosé María Suárez Núñez, s/n, Santiago de Compostela, E-15782, Spain

E-mail: collar@uchicago.edu, pcoloma@ific.uv.es, ivan.esteban@fqa.ub.edu, paola.ferrario@dipc.org, jjgomezcadenas@dipc.org, maria.gonzalez-garcia@stonybrook.edu, francesc.monrabal@dipc.org 
AbStract: The European Spallation Source (ESS), presently well on its way to completion, will soon provide the most intense neutron beams for multi-disciplinary science. Fortuitously, it will also generate the largest pulsed neutrino flux suitable for the detection of Coherent Elastic Neutrino-Nucleus Scattering ( $\mathrm{CE} \nu \mathrm{NS})$, a process recently measured for the first time at ORNL's Spallation Neutron Source. We describe innovative detector technologies maximally able to profit from the order-of-magnitude increase in neutrino flux provided by the ESS, along with their sensitivity to a rich particle physics phenomenology accessible through high-statistics, precision $\mathrm{CE} \nu \mathrm{NS}$ measurements.

Keywords: Neutrino Detectors and Telescopes (experiments), Beyond Standard Model, Electroweak interaction

ArXiv EPrint: 1911.00762 


\section{Contents}

1 Introduction 1

2 The ESS as a neutrino source: comparison to the SNS 3

3 Detector technologies $\quad 7$

$\begin{array}{lll}3.1 & \text { Cryogenic }(77 \mathrm{~K}) \text { undoped CsI scintillator array } & 7\end{array}$

3.2 Low-background CCD arrays with single-electron threshold 10

$\begin{array}{ll}3.3 & \text { High-pressure gaseous xenon chambers } \\ & 12\end{array}$

$\begin{array}{lll}3.4 & \text { Low-threshold, multi-kg p-type point contact germanium detectors } & 13\end{array}$

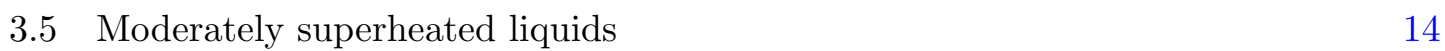

4 Physics reach $\quad 16$

$\begin{array}{lll}4.1 & \text { Parameters and assumptions used in calculations } & 16\end{array}$

$\begin{array}{lll}4.2 & \text { Non-standard neutrino interactions } & 19\end{array}$

4.3 Weak mixing angle and neutrino charge radii 24

$\begin{array}{lll}4.4 & \text { Neutrino magnetic moment } & 26\end{array}$

$\begin{array}{lll}5 & \text { Conclusions } & 27\end{array}$

\section{Introduction}

Low-energy neutrinos can scatter off the atomic nucleus as a whole, via the weak neutral current. During this process the initial and final states of the nuclear target are indistinguishable, permitting a coherent contribution from all nucleons. The net result is a drastic enhancement to the cross-section for this type of neutrino interaction, roughly proportional to the square of the number of neutrons present in the target nucleus. The single observable from this so-called Coherent Elastic Neutrino-Nucleus Scattering (CE $\nu \mathrm{NS}$ ) is a recoiling nucleus, which generates a signal in the few $\mathrm{keV}$ to sub-keV energy range, difficult to reach with most contemporary radiation detectors. An additional obstacle to $\mathrm{CE} \nu \mathrm{NS}$ detection is the limited number of suitable neutrino sources, both sufficiently intense in yield, and low enough in neutrino energy so the coherence condition can be satisfied (that is, $|Q|<1 / R$, where $|Q|$ is the momentum transfer and $R$ is the radius of the nucleus).

Perhaps the most convenient of the available possibilities are the neutrinos produced following the decay at rest of positive pions at spallation sources. The first advantage these provide is the generation of nuclear recoils as energetic as is allowed by the coherence condition, facilitating their detection [1]. Additionally, the pulsed beam timing that is typically characteristic of this type of source reduces the impact of steady-state backgrounds able to mask the signal, in proportion to a small duty factor. CE $\nu$ NS was experimentally demonstrated by the COHERENT experiment [2] forty-three years following its theoretical 


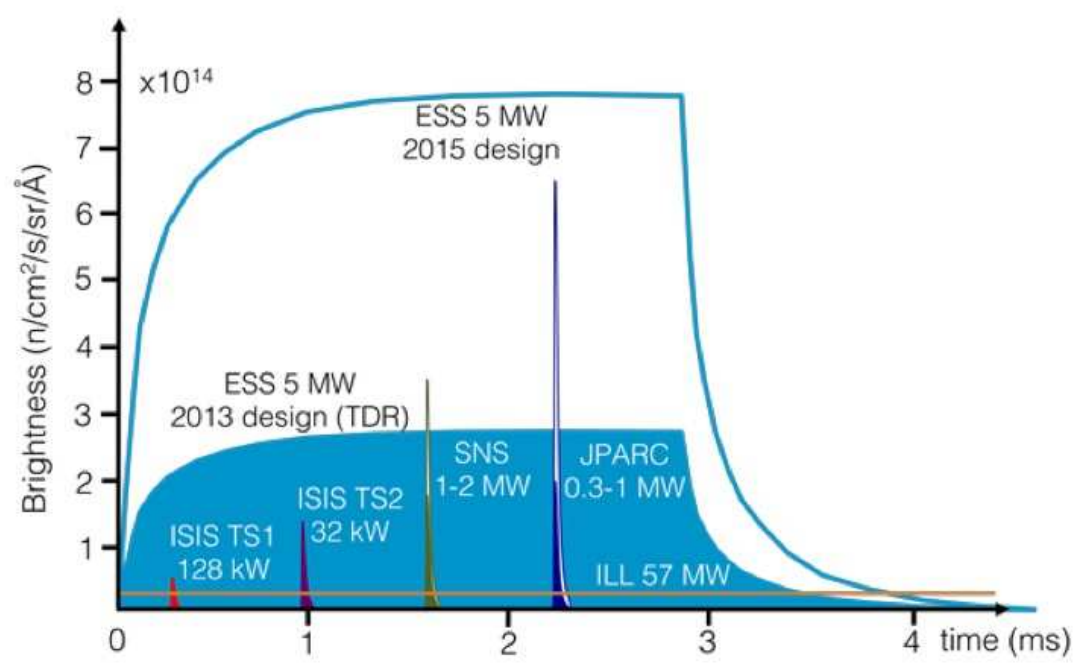

Figure 1. (Source: ESS) Neutron production from existing and planned spallation sources. The nominal SNS power is $1 \mathrm{MW}$ at proton energy $1 \mathrm{GeV}$, with a plan to reach $2 \mathrm{MW}$ by 2026 . The ESS power will be $5 \mathrm{MW}$ at $2 \mathrm{GeV}$ circa 2023 , with the ability to further upgrade. Differences in the duration of the protons-on-target (POT) pulse are visible in the figure. The ESS will generate an increase in neutron brightness by a factor 30-100 with respect to previous spallation sources, and an order of magnitude larger neutrino yield than the SNS.

description [3], using the presently most intense Spallation Neutron Source (SNS), sited at the Oak Ridge National Laboratory, U.S.A.. A low-background $14.6 \mathrm{~kg}$ CsI[Na] scintillator with optimal characteristics for this goal $[4,5]$ was employed as the detecting medium.

Besides a miniaturization of neutrino detectors, and any future technological applications that might bring, $\mathrm{CE} \nu \mathrm{NS}$ has been proposed as a new tool for the study of fundamental neutrino properties. Phenomenological work following the first $\mathrm{CE} \nu \mathrm{NS}$ measurement has generated improved bounds on non-standard neutrino interactions (NSI) [6-16], as well as contributions to the study of nuclear structure [17-21]. The possibility of constraining our present knowledge of neutrino electromagnetic properties [22-26] and of the weak mixing angle [27-29], together with the potential to search for sterile neutrinos [30, 31], or for new types of dark matter particles [32-34], has also been examined.

In this work we describe the opportunity to perform high-statistics CE $\nu$ NS measurements provided by the upcoming European Spallation Source (ESS) sited in Lund, Sweden. The ESS will combine the world's most powerful superconducting proton linac with an advanced hydrogen moderator, generating the most intense neutron beams for multidisciplinary science (figure 1). It will also provide an order of magnitude increase in neutrino flux with respect to the SNS. This will facilitate $\mathrm{CE} \nu \mathrm{NS}$ measurements not limited in their sensitivity to new physics (NP) by poor signal statistics, while still employing nonintrusive, compact (few $\mathrm{kg}$ ) neutrino detectors, able to operate without interference with ESS neutron activities.

This manuscript is organized as follows. Section 2 describes the characteristics of the ESS as a neutrino source for CE $\nu$ NS, establishing a positive comparison with the SNS 
in all aspects involved, while also delineating the ESS site characterization activities that will be necessary to confirm this strong potential. Section 3 briefly describes a number of state-of-the-art nuclear recoil detector technologies maximally able to exploit the opportunity that the ESS represents. Section 4 discusses the physics reach provided by the combination of this source and these detectors, on a number of phenomenological fronts probing for deviations from the Standard Model (SM). Our conclusions are presented in section 5. Chief among those is the unique opportunity provided by the ESS to perform precision studies of $\mathrm{CE} \nu \mathrm{NS}$, for which the statistics of the neutrino signal will contribute a subdominant uncertainty.

\section{The ESS as a neutrino source: comparison to the SNS}

At spallation sources, both $\pi^{+}$and $\pi^{-}$are produced in proton-nucleus collisions in the target. While the resulting $\pi^{-}$are efficiently absorbed by nuclei before they can decay, the produced $\pi^{+}$lose energy as they propagate in the target and will eventually decay at rest (DAR) into $\pi^{+} \rightarrow \mu^{+} \nu_{\mu}$, followed in close spatial vicinity (within $\sim 0.2 \mathrm{~g} / \mathrm{cm}^{2}$ ) by $\mu^{+} \rightarrow e^{+} \nu_{e} \bar{\nu}_{\mu}$. Three neutrino flavors with essentially identical CE $\nu \mathrm{NS}$ cross section [35], are therefore engendered for each $\pi^{+}$created. Being the result of a two-body decay, the $\nu_{\mu}$ flux is monochromatic: $E_{\nu_{\mu}}=\left(m_{\pi}^{2}-m_{\mu}^{2}\right) /\left(2 m_{\pi}\right) \simeq 29.7 \mathrm{MeV}$, where $m_{\pi}$ and $m_{\mu}$ refer to the pion and muon masses, respectively. Conversely, the $\nu_{e}$ and $\bar{\nu}_{\mu}$ fluxes follow a continuous distribution at energies $E_{\nu_{e}, \bar{\nu}_{\mu}}<m_{\mu} / 2 \simeq 52.8 \mathrm{MeV}$. Normalized to one, they read:

$$
\begin{aligned}
& f_{\bar{\nu}_{\mu}}\left(E_{\nu}\right)=\frac{64}{m_{\mu}}\left[\left(\frac{E_{\nu}}{m_{\mu}}\right)^{2}\left(\frac{3}{4}-\frac{E_{\nu}}{m_{\mu}}\right)\right], \\
& f_{\nu_{e}}\left(E_{\nu}\right)=\frac{192}{m_{\mu}}\left[\left(\frac{E_{\nu}}{m_{\mu}}\right)^{2}\left(\frac{1}{2}-\frac{E_{\nu}}{m_{\mu}}\right)\right] .
\end{aligned}
$$

Since the lifetime of the muon is much longer than that of the pion, the monochromatic component is usually referred to as the prompt contribution to the flux, as opposed to the delayed contributions from $\mu^{+}$decay. For reference, the flux spectra is shown in figure 2, for the three components separately.

Besides the obvious gain in statistics with respect to other neutrino sources, the use of neutrinos from pion decay at rest presents a clear advantage: the energy dependence of the flux is well-known in this case, and there is only room for systematic uncertainties affecting its normalization. This contrasts with the large uncertainties associated to conventional neutrino beams, where neutrinos are produced in the decay of pions and kaons in flight, and the determination of the neutrino spectral shape relies on Monte Carlo simulations.

The described energy spectrum for neutrinos from spallation sources is rather independent from the characteristics of the proton beam, and therefore it is very similar for SNS and ESS. There are however, important differences in intensity and temporal structure that we describe next.

The ESS is scheduled to reach its design power of $5 \mathrm{MW}$ and goal proton energy of $2 \mathrm{GeV}$ by 2023, with a neutron user program commencing soon after [36]. First protons-ontarget (POT) are imminent, expected for 2021 at a reduced $0.5 \mathrm{GeV}$. The power delivered 


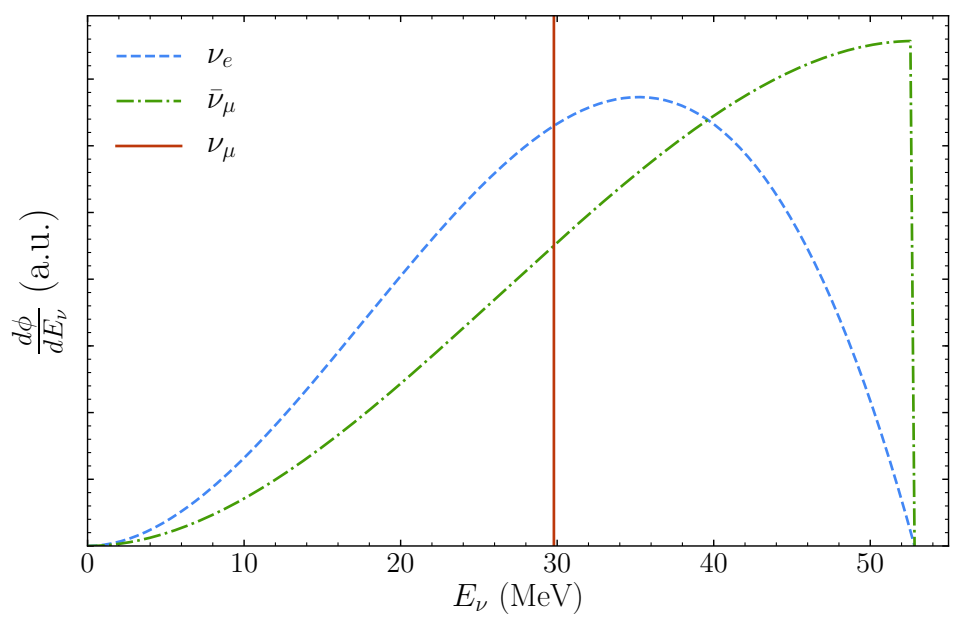

Figure 2. Neutrino flux spectra expected from pion DAR, in arbitrary units (a.u.), as a function of the neutrino energy in $\mathrm{MeV}$. The three components of the flux are shown separately as indicated by the legend. The distributions have been normalized to one.

by a spallation source can be regarded as the product of proton current, and energy per proton. From this perspective, the proton energy of $\sim 1 \mathrm{GeV}$ and nominal $1 \mathrm{MW}$ design power of the SNS implies an increase in average proton current at the ESS by a factor of 2.5 , for a total of $2.8 \times 10^{23}$ POT per calendar year. Both ESS and SNS operate on a scheduled 5,000 hours of beam delivery per year, the downtime being reserved for facility and accelerator maintenance.

The decay-at-rest (DAR) neutrino yield is expected to increase rapidly with proton energy at spallation sources. A dedicated calculation of the ESS neutrino yield [37, 38] modified the LAHET (Bertini model) Monte Carlo code [39] to include experimental data on pion production in the $0.5-2.5 \mathrm{GeV}$ proton energy range. This reference work found an increase by a factor 4.4 in neutrino yield for a tungsten target, in going from the nominal $0.94 \mathrm{GeV}$ proton energy of the SNS [2] to the $2 \mathrm{GeV}$ of a completed ESS. In particular, the probability of inducing a second pion-generating nuclear interaction per initial proton is predicted to climb rapidly with proton energy, as does double-pion production per interaction, and the ratio of pion decays to captures [37]. A comparison of theoretical predictions with experimental data for a number of neutrino cross section measurements assigns a modest uncertainty to these DAR neutrino production calculations, validating them [40].

Nevertheless in this work we perform our own simulations as an additional test of the neutrino production rates at the ESS. In particular we have performed simulations with MCNPX [41], GEANT4 [42], and FLUKA [43] shown in figure 3. While MCNPX was used for the baseline design and target optimization of the ESS [36, 44], recent comparisons with GEANT4 qualify the latter as a comparable tool, at least from the point of view of neutron production and transport [45]. Figure 3 shows a typically fair agreement between all codes and the calculated neutrino production in $[37,38]$, for a proton energy of $0.94 \mathrm{GeV}$. This agreement is preserved at $2 \mathrm{GeV}$ for most MCNPX 2.7.0 intranuclear cascade and evaporation model combinations, but not for the GEANT4 options tested. A large dispersion in 


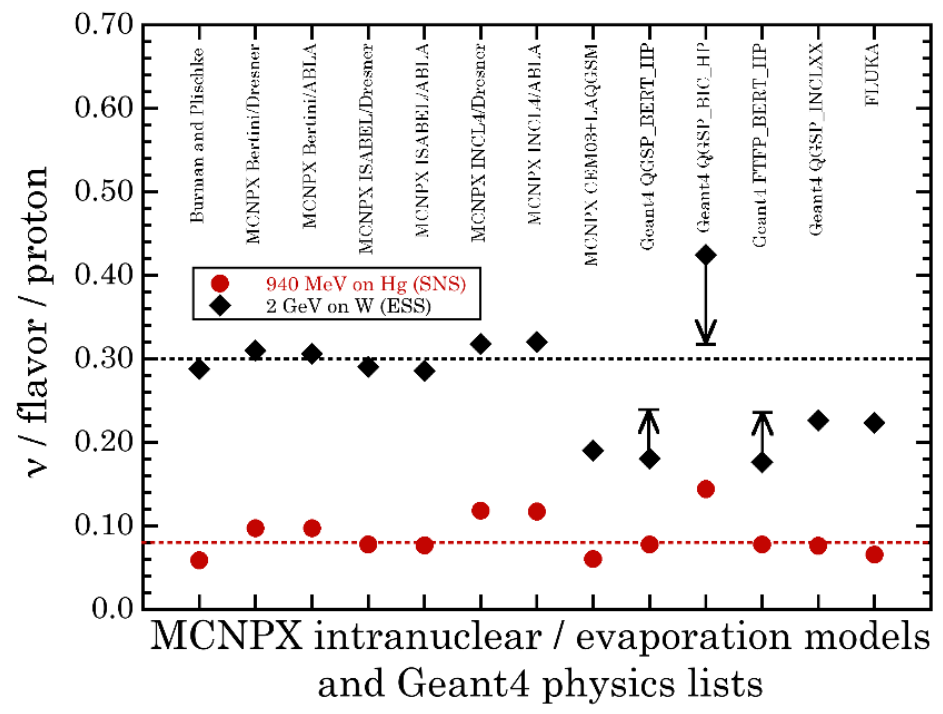

Figure 3. Neutrino yields for the SNS mercury and ESS tungsten targets, as a function of simulation package adopted. The first column shows predictions from a dedicated calculation [37, 38], validated against neutrino cross section measurements [40]. For three physics lists in common with [48], arrows attempt a correction based on the HARP-CDP $\pi^{+}$production cross-section for $2.2 \mathrm{GeV}$ protons on Ta. The horizontal red line marks the value used for the $\mathrm{CE} \nu \mathrm{NS}$ measurement at the SNS [2], whereas the black line shows the value adopted here for the ESS.

GEANT4 pion yield predictions has been noticed before, during benchmarks using the most recent hadroproduction measurements by the HARP [46] and HARP-CDP [47] collaborations: experimental pion production cross-sections for $2.2 \mathrm{GeV}$ protons striking $\mathrm{Ta}$ and $\mathrm{Pb}$ targets are over- or under-estimated by different GEANT4 physics lists [48-50], following a pattern similar to that in figure 3. In addition to this, a comparison with HARP-CDP data [51] indicates that $\pi^{+}$production by $0.8 \mathrm{GeV}$ protons is slightly underestimated by the LAHET parametrization in $[37,38]$.

Based on these considerations, we tentatively adopt a yield of 0.3 neutrinos of each flavor $\left(\nu_{\mu}, \bar{\nu}_{\mu}, \nu_{e}\right)$ per proton for a ESS operating at $2 \mathrm{GeV}$, a considerable improvement of a factor 3.75 over the corresponding figure of 0.08 at the SNS [2] (but a bit more conservative than the 4.4 increase factor found in refs. $[37,38])$. In combination with the increased ESS proton current mentioned above, this results in an expected $8.5 \times 10^{22}$ neutrinos per flavor per year, an order of magnitude higher than the equivalent of $9.2 \times 10^{21}$ from a reference $1 \mathrm{MW}, 0.94 \mathrm{GeV}$ SNS [52]. On the subject of neutrino flux verification, a proposal to use a small $\left(1 \mathrm{~m}^{3} \mathrm{D}_{2} \mathrm{O}\right)$ charged-current detector to independently measure the neutrino yield of the SNS [52-54] may be worth replicating at the ESS.

A second difference between SNS and ESS is in their proton beam pulse timing: $60 \mathrm{~Hz}$ of $1 \mu$ s-wide POT spills at the SNS, vs. $14 \mathrm{~Hz}$ of $2.8 \mathrm{~ms}$ spills at the ESS (figure 1). Naively, beam timing at the SNS would seem much more favorable for steady-state background reduction in $\mathrm{CE} \nu \mathrm{NS}$ detectors [55]. The SNS duty factor is $6 \times 10^{-4}$, accounting for $10 \mu \mathrm{s}$ after POT pulses to encompass the delayed $\bar{\nu}_{\mu}, \nu_{e}$ neutrino emission from muon decay [2]: 
this duty factor becomes $4 \times 10^{-2}$ at the ESS. However, steady-state backgrounds can be accurately characterized during the long anti-coincident periods between beam spills, to then be subtracted from POT-coincident windows that in addition contain the CE $\nu \mathrm{NS}$ signal. In the absence of beam-related backgrounds, this results in a residual departing from zero proportionally to the $\mathrm{CE} \nu \mathrm{NS}$ signal, with statistical error bars partly defined by the steady-state background level achieved [2]. The signal-to-background figure of merit is then seen to be slightly favorable for the ESS (the square root of the ratio of the ESS and SNS duty factors is $10 \%$ smaller than the factor of nine increase in CE $\nu$ NS signal rate from a larger ESS neutrino flux).

Regardless of this minor advantage, the main attraction of the ESS is in the accumulation of $\mathrm{CE} \nu \mathrm{NS}$ signal statistics ten times faster than at the SNS. An example of the relevance of this aspect can be found in the sluggish signal growth rate for the $14.6 \mathrm{~kg}$ CsI[Na] detector at the SNS: some $\sim 300$ events in four calendar years, for a heavy-nuclei target with a large $\mathrm{CE} \nu \mathrm{NS}$ cross-section. In contrast to this, the similarly compact detectors presented in section 3 aim to register up to thousands of events per year at the ESS (table 1), through a combination of increased neutrino flux, and improved detector performance.

Related to the timing consideration above, at the ESS there will be no temporal separation possible between neutrino flavors, with prompt $\nu_{\mu}$ and delayed $\bar{\nu}_{\mu}, \nu_{e}$ becoming indistinguishable due to the very long $(2.8 \mathrm{~ms})$ beam spills. At the ESS, a partial discrimination is nevertheless possible using recoil energy spectrum, which reduces the impact of this limitation for most NP searches, as will be discussed section 4.2. There are however a few exceptions where a distinction between prompt and delayed signals is mandatory, such as the search schemes described in [31,34]. As shown by COHERENT [2] an opportunity exists at the SNS to (partially) isolate prompt from delayed neutrino components via timing, but only for detectors sited in a small area of the "neutrino alley" [2], of roughly $10 \times 1$ $\mathrm{m}^{2}$. This limitation is due to a large gradient in prompt neutron background measured along the length of the alley (five orders of magnitude over $25 \mathrm{~m}[5,56,57]$ ), and safety restrictions on corridor width encumbrance. The end of the SNS alley farthest from target (28 m) offers additional space for detectors requiring ancillary equipment such as cryogenic and purifier stages for liquid noble targets. However, the prompt neutron background from the nearby beam line makes $\nu_{\mu}$ detection entirely unfeasible at this location $[56,57]$, further reducing the available SNS neutrino flux by one third.

Still on the subject of space availability, a strong underlying assumption in this work is that a ESS location will be found that is similar to the SNS neutrino alley, in its optimal proximity to target, shielding against prompt neutrons, and absence of interference with neutron activities. Specifically, an instantaneous (i.e., POT-coincident) flux of less than $\sim 2 \times 10^{-3}$ neutrons $/ \mathrm{cm}^{2} \mathrm{~s}\left(E_{n}>1 \mathrm{MeV}\right)$ will be required at the ESS to achieve a negligible beam-coincident neutron background level, similar to that in [2]. The lessons learned in this respect from SNS experimentation (neutron leakage from beam line, influence of materials and voids in the line of sight to the target monolith, beneficial effect of basement overburden against skyglow) can be used to minimize the scouting for such locations. Initial investigations of the availability of ESS sites viable for $\mathrm{CE} \nu \mathrm{NS}$ studies indicate that 
$22 \mathrm{~m}^{2}$ of unallocated space $15-23 \mathrm{~m}$ from target exists, with 5 meters of steel or iron and a minimum of 6 meters of magnetite-loaded heavy concrete in the line of sight to target. The density of this concrete is twice that of the compacted limestone gravel between target monolith and neutrino alley at the SNS. Dedicated neutron background measurements and simulations will be necessary to confirm the suitability of this and other ESS locations for the activities described next.

\section{Detector technologies}

In this section we present a number of detector technologies sensitive to low-energy $\left(\lesssim 1 \mathrm{keV}_{\mathrm{nr}}\right)$ nuclear recoils, aligned to profit from the increased neutrino flux at the ESS. Some possess characteristics able to maximize the physics reach to certain physics scenarios, and in combination they can further boost the attainable sensitivity, as we illustrate in section 4. The common origin of these techniques is in the fields of dark matter detection, and double-beta decay searches. A best-effort in background abatement already made for these detectors can be reinvested for $\mathrm{CE} \nu \mathrm{NS}$ at the ESS. For convenience, the characteristics of the detectors considered, and expected signal and background rates assumed in the sensitivity studies presented in section 4 are summarized in table 1 .

\subsection{Cryogenic $(77 \mathrm{~K})$ undoped CsI scintillator array}

Pure CsI operated at liquid nitrogen temperature exhibits a light yield in the range 80-125 photons per $\mathrm{keV}$ [58-66]. This is at the maximum theoretical efficiency in the conversion of energy deposition to scintillation, with almost all electron-holes created in the crystal recombining radiatively (figure $4[67,68]$ ). This behavior is remarkable, as the number of information carriers generated per unit deposited energy is just a factor of three smaller than for Ge and Si semiconductor detectors. Provided that a good quantum efficiency (QE) in the light sensor is achieved, this can facilitate the detection of low-energy signals, with optimal resolution. The potential of cryogenic CsI for neutrino and dark matter detection has attracted attention [61-63, 65, 69]. This material further improves on the virtues of $\mathrm{CsI}[\mathrm{Na}]$ for $\mathrm{CE} \nu \mathrm{NS}$ [4], by increasing its light output by a factor of $\gtrsim 2$ (figure 4 ).

In order to establish the feasibility of using cryogenic CsI at the ESS, two cryostats have been developed at the University of Chicago. One is dedicated to large-area avalanche photodiode (LAAPD) [58-60, 76, 79] readout of CsI crystals at $80 \mathrm{~K}$. The second is reserved for Hamamatsu R8520-506 [80] photomultiplier (PMT) use at $108 \mathrm{~K}$. This ongoing R\&D is of crucial importance to establish the method: for instance, the quenching factor $(\mathrm{QF})$ for low-energy nuclear recoils (NRs) in pure cryogenic CsI has not been measured before. Our preliminary findings at $108 \mathrm{~K}$ (figure 5) indicate that it is of $\mathrm{O}(10) \%$, comparable to that for $\mathrm{CsI}[\mathrm{Na}]$ at room temperature [71]. An anomalous increase of the QF for alphas in CsI at low temperature [61, 62], and the possible presence of coadjutant low-energy processes such as the Migdal effect [81], are a reminder of the need for a full QF characterization. We temporarily adopt an energy-independent value of $10 \%$ (table 1 ).

As part of the upgrade of the Belle-II CP-violation experiment, novel wavelength shifters (nanostructured organosilicon luminophores, NOL $[78,82-86]$ ) have been used to 


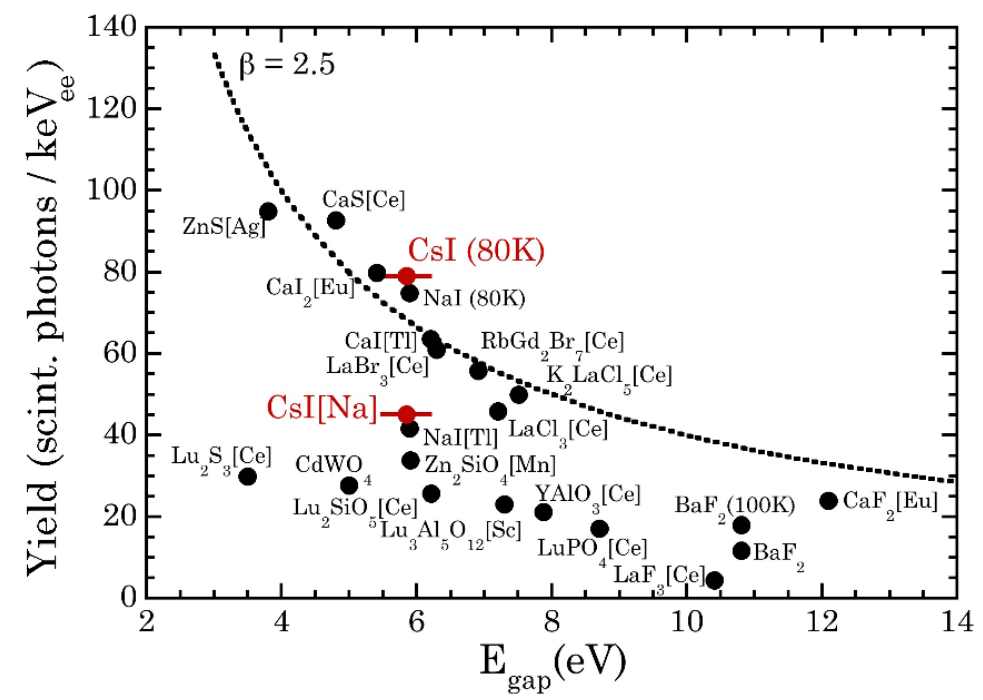

Figure 4. Light yield of scintillators and phosphors as a function of bandgap, adapted from [68]. A dotted line indicates a model-dependent maximum light yield [68]. Red dots mark our measurements for cryogenic CsI, in agreement with [63,65], and for room temperature CsI[Na] [4]. Yields of up to $125 \mathrm{ph} / \mathrm{keV}_{e e}$ have been claimed for other CsI stock [59,60]. A CsI bandgap range is indicated by horizontal error bars [70].

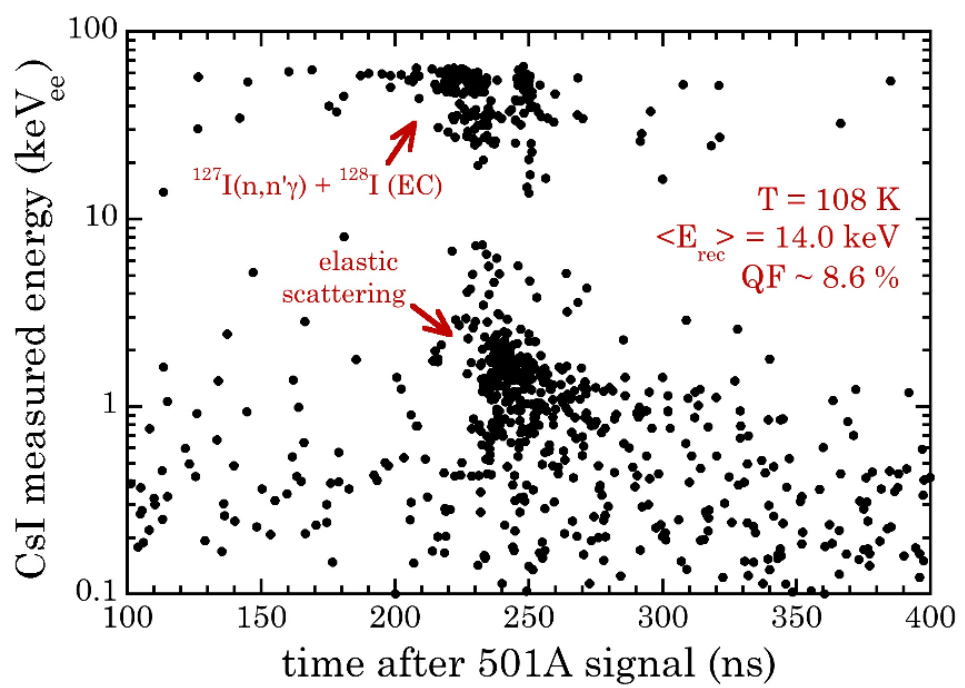

Figure 5. First measurement of nuclear recoil quenching factor (QF) for cryogenic CsI. The figure displays the measured electron-equivalent energy $\left(\mathrm{keV}_{e e}\right)$ deposited by $14 \mathrm{keV} \mathrm{Cs}$ and I nuclear recoils induced by $2.2 \mathrm{MeV}$ neutron scattering. The reader is referred to a similar figure 2 in [71], for more details on the methodology employed in QF determination.

reach a quantum efficiency $\mathrm{QE} \gtrsim 80 \%$ during avalanche photodiode (APD) monitoring of room-temperature CsI $[73,74]$. Figure 6 displays a satisfactory first demonstration of NOL performance at cryogenic temperature, using our LAAPD cryostat. The light-detection QE obtained following wavelength shifting of the $340 \mathrm{~nm}$ cryogenic CsI emissions [58, 64] to $590 \mathrm{~nm}$ is a factor of three larger than possible with existing cryogenic PMT photocath- 


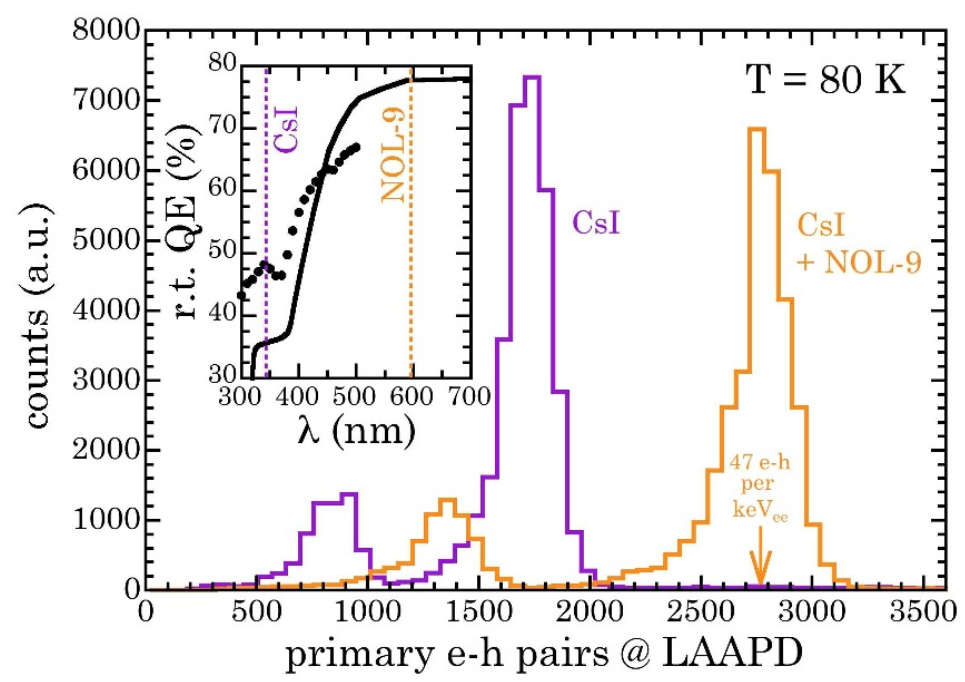

Figure 6. Response of a $3.2 \mathrm{~cm}^{3}$ cryogenic CsI crystal to $59.5 \mathrm{keV}{ }^{241} \mathrm{Am}$ gammas, seen by a $1.3 \times 1.3 \mathrm{~cm}^{2}$ LAAPD [72], with and without a NOL-9 wavelength shifter plate [73, 74]. Escape peaks appear at $55.5 \mathrm{keV}$ and $29.7 \mathrm{keV}$. LAAPD gain and noise $(\mathrm{G}=1,060,4 \mathrm{eV} \mathrm{rms})$ were monitored via concurrent silicon surface irradiation with ${ }^{55}$ Fe x-rays [59, 60]. Plasma effects [75] and QE reduction with temperature [76] were considered. Inset: available room-temperature (r. t.) QE data from the LAAPD manufacturer (dots), and for a generic Si APD (line) [77], together with wavelengths of CsI emission $(80 \mathrm{~K},[58,64])$ and of NOL-9 r. t. luminescence [78]. The increase in photon detection is as expected from an efficient wavelength shift. A $1 \mathrm{keV}$ nuclear recoil with $\mathrm{QF}=10 \%$ will generate a 4.7 primary e-h signal, above LAAPD threshold (see text).

odes [80]. We observe an excellent long-term stability in the performance (electronic noise, internal gain, light yield) of the CsI+NOL+LAAPD combination at $80 \mathrm{~K}$.

LAAPDs with surface area $45 \mathrm{~cm}^{2}$, when operated at $77 \mathrm{~K}$, exhibit stable internal gains in excess of 1,000, and a reduced leakage current leading to a light-detection threshold of approximately four photons [87], i.e., four primary electron-hole (e-h) pairs at the LAAPD (figure 6) prior to avalanche amplification. The demonstrated combination of a high light yield (figure 4), optimal QE for its detection (figure 6), few-photon LAAPD threshold [87], and a quenching factor of order $10 \%$ (figure 5), adds up to a predicted sensitivity to $\sim 1 \mathrm{keV}$ nuclear recoils in this hybrid cryogenic detector. This can be contrasted with the $\sim 10 \mathrm{keV}$ obtained with super-bialkali PMT readout of room temperature CsI[Na] during the first $\mathrm{CE} \nu \mathrm{NS}$ measurement $[2,5]$. Furthermore, $\mathrm{CsI}[\mathrm{Na}] \mathrm{NR}$ signal acceptance was limited there to $65 \%$, as a result of cuts to reject Cherenkov light emission in the PMT glass envelope. This is a dominant source of low-energy background that is absent from LAAPDs. Taking the effect of signal acceptance into account, figure 7 shows that an increase by a factor of eight in CE $\nu \mathrm{NS}$ signal rate per unit CsI mass can be obtained from this alternative cryogenic approach. The steady-state background adopted in table 1 is that achieved for CsI $[\mathrm{Na}]$ at the SNS $[2,5]$, with the estimated Cherenkov contribution subtracted.

The presently envisioned design of a ESS cryogenic CsI detector is a small array of four crystals of individual dimensions $5 \times 5 \times 50 \mathrm{~cm}^{3}$, each read out by two $25 \mathrm{~cm}^{2}$ LAAPDs, 


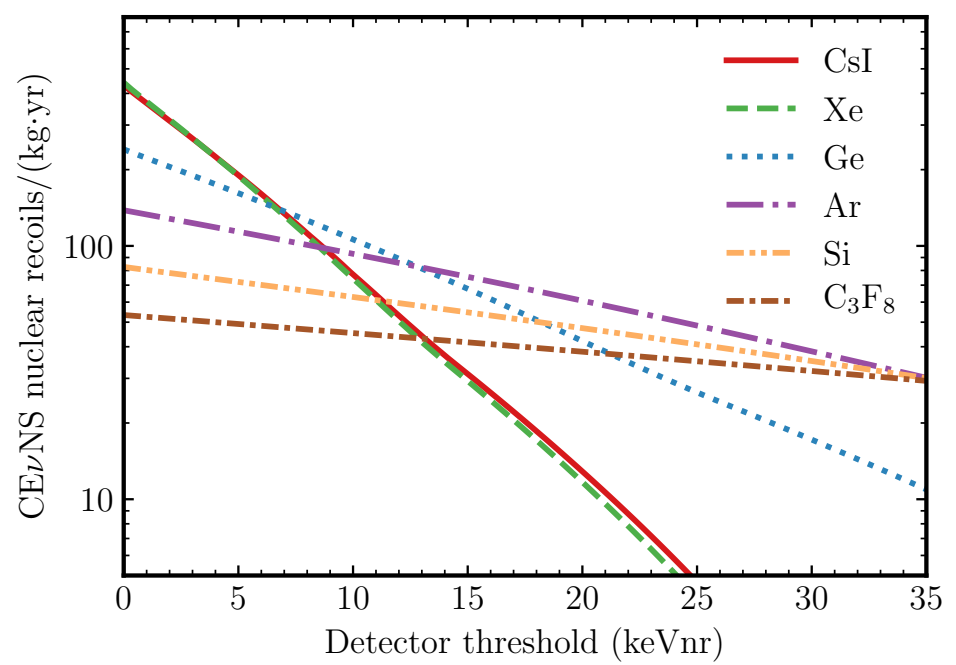

Figure 7. Expected integrated $\mathrm{CE} \nu \mathrm{NS}$ rate above nuclear recoil threshold, $20 \mathrm{~m}$ away from the ESS target, for all detector materials considered in this work. All technologies considered for use at the ESS have thresholds at or below $1 \mathrm{keV}_{\mathrm{nr}}$.

for a total mass of $22.5 \mathrm{~kg}$. This compact detector is expected to provide approximately $8,000 \mathrm{CE} \nu \mathrm{NS}$ events per year at the ESS (figure 7), a signal throughput two orders of magnitude faster than during the first $\mathrm{CE} \nu \mathrm{NS}$ detection [2]. Three hundred CsI crystals of these dimensions are in storage at the University of Chicago [88], left from the KTeV experiment, allowing for an eventual detector mass upgrade.

\subsection{Low-background CCD arrays with single-electron threshold}

High resistivity, $\approx$ mm-thick silicon Charge Coupled Devices (CCDs) have been recently demonstrated as an effective detector technology for the search of rare events from dark matter [89-91] and neutrino [92] interactions. Efforts are ongoing for the construction of DAMIC-M [93], a kg-size detector to be installed at the Laboratoire Souterrain de Modane (LSM) in France. To achieve a leading dark matter sensitivity, DAMIC-M will require

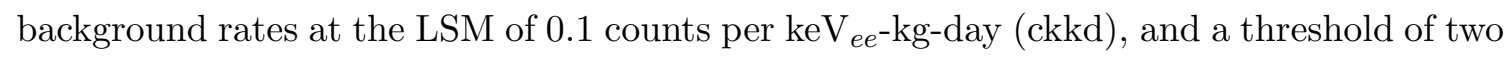
ionized electrons (corresponding to a few eV in ionization energy). This ultra-low energy threshold and background rate can be leveraged for $\mathrm{CE} \nu \mathrm{NS}$ detection. As discussed in section 4 , the sensitivity to a finite neutrino magnetic moment via $\mathrm{CE} \nu \mathrm{NS}$ becomes maximal at the lowest recoil energies. A detector with characteristics like DAMIC-M in threshold and energy resolution would be ideal for this $\mathrm{CE} \nu \mathrm{NS}$ application. In the following we will use DAMIC-M as an example for a kg-size CCD-based silicon detector to be installed at the ESS. DAMIC-M will be nearing completion of its exposure at the LSM by the time the ESS reaches its full $5 \mathrm{MW}$ power.

The beam timing characteristics of the ESS involve a data-acquisition approach different from that of a dark matter search. For a typical single pixel read-out time of $10 \mu \mathrm{s}$ the total read-out time for a Mpixel device would be several seconds, too slow for the ESS beam-spill frequency. We thus plan to perform "hardware-binning" (namely to sum the 


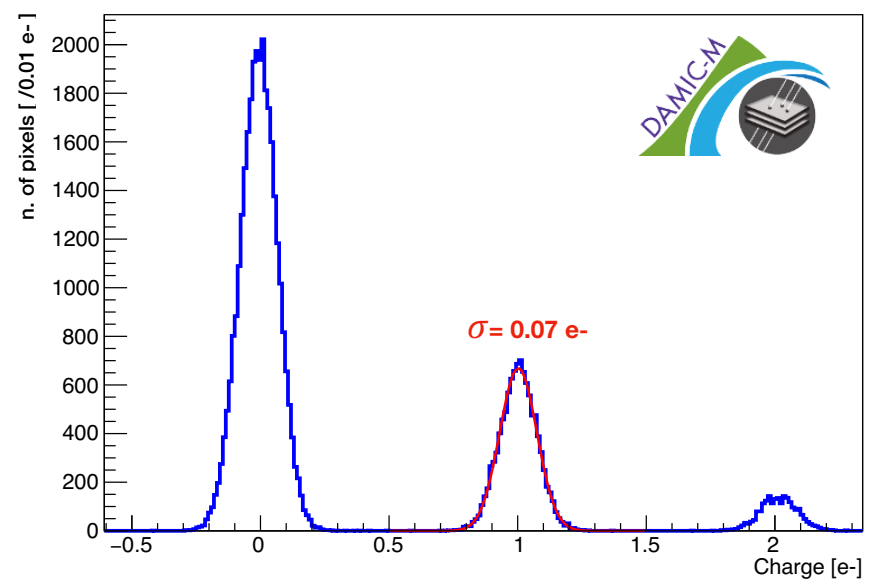

Figure 8. (From [94]) First demonstration of single-electron sensitivity in DAMIC-M CCDs, using a Skipper readout. An average ionization energy deposition of $3.77 \mathrm{eV}$ is necessary for the creation of a $1 \mathrm{e}^{-}$signal in silicon [95].

signal of several pixels before read-out), a technique which improves read-out speed and read-out noise with only minimal loss of event information. Hardware binning has been successfully employed by DAMIC@SNOLAB [89]. The number of binned pixels that can be readout given the ESS beam-spill frequency of $14 \mathrm{~Hz}$ will depend on the number of amplifiers integrated in the CCD, with about 7000 binned pixels per amplifier per beam spill. Taking the current design of a DAMIC-M CCD as example $(6 \mathrm{k} \times 6 \mathrm{k}$ pixels, each $15 \mu \mathrm{m}$ $\times 15 \mu \mathrm{m}$, readout by four amplifiers), 14,000 binned pixels can be readout per spill when accounting for identical $2.8 \mathrm{~ms}$ POT-coincident and -anticoincident exposures per beam spill (the second used to characterize steady-state backgrounds). This can be achieved by a $50 \times 50$ binning (corresponding to a $750 \mu \mathrm{m} \times 750 \mu \mathrm{m}$ sensitive area) or any other suitable combination of rows and columns summing a total of 2500 pixels. No hardware modifications are required for this purpose. Future CCDs could be better optimized for ESS timing by incorporating more amplifiers for faster read-out.

Instrumented with a Skipper readout [96], DAMIC-M will feature single-electron sensitivity. DAMIC-M has recently demonstrated such sensitivity using $1 \mathrm{k} \times 6 \mathrm{k}$ CCDs to achieve a resolution of less than 0.1 electrons [94], as shown in figure 8. This is accomplished by using a novel approach to data acquisition, wherein the charge in a single pixel is measured non-destructively multiple $(\mathrm{N})$ times resulting in a reduction of the overall read-out noise by a factor of $\sqrt{N}$. While this certainly could not be implemented for every pixel at the ESS, an adaptive read-out mode wherein a pixel is measured multiple times only if it meets a set of selection criteria may provide improved energy threshold with minimal loss of exposure.

As mentioned, DAMIC-M is designed to reach a 0.1 ckkd internal radioactive background, far lower than other solid-state detectors of the same scale. The steady-state background for a silicon CCD detector at the ESS is therefore expected to be cosmic-ray associated, and dominated by muon-induced neutrons in lead shielding, which can be re- 
jected using an active veto. Instead of discarding individual events, as is done for faster detectors, entire veto-coincident individual CCD exposures must be rejected. Accounting for a $100 \mathrm{~Hz}$ muon veto trigger rate under minimal overburden, and for the ESS beamtiming characteristics, we estimate that this can be achieved with just $\sim 28 \%$ dead time, while rejecting $>99.9 \%$ of muon-induced backgrounds. These expectations are solidly based on the germanium PPC experimentation at shallow depth described below. In the absence of a measurement of background level at a shallow site, we presently assume that the background rates at the ESS will be a factor of ten higher than those expected by DAMIC at the LSM, and thus adopt a background level of 1 ckkd in the sensitivity estimates of section 4 . The $15 \mathrm{ckkd}$ at $0.18 \mathrm{keV}_{e e}$ obtained in a shallow site with less-radioclean PPCs (figure 10) is a realistic upper bound.

The quenching factor for DAMIC silicon CCDs has been measured down to $0.7 \mathrm{keV}_{\mathrm{nr}}$ [97], using a photoneutron source [98]. A new measurement down to a considerably lower energy is planned using the same technique, while profiting from the recent progress in single-electron sensitivity. As emphasized in [71], a precise knowledge of this quenching factor will be necessary to fully exploit the sensitivity of CE $\nu$ NS detectors to NP. The incipient but rapid development of new germanium CDDs [99] is worth mentioning as a possible future upgrade of this technique, as those can provide a higher $\mathrm{CE} \nu \mathrm{NS}$ signal rate (figure 7), and a more favorable quenching factor.

\subsection{High-pressure gaseous xenon chambers}

High-pressure gaseous detectors such as NEXT [100], sensitive to both primary scintillation (S1) and electroluminescent amplification of charge ionization (S2), provide excellent background discrimination, and optimal energy resolution (figure 9). This technology is not impacted by charge trapping and delayed release at the liquid-gas interface of dual-phase liquid noble gas detectors $[101,102]$. This process limits dual-phase detector sensitivity to low-energy signals at shallow depth sites dominated by frequent large-energy depositions by cosmic rays [103], making NEXT-like detectors more suitable for operation at a location with negligible overburden, like the ESS.

Furthermore, in principle the ability to trigger data acquisition using a POT-coincident logic signal can eliminate the need to detect primary scintillation light at the ESS, resulting in a reduced energy threshold. By using electroluminescence amplification, signals as low as 1-2 ionized electrons can be detected. This reduces the expected energy threshold to less than $0.2 \mathrm{keV}_{e e}$. In addition to this, gaseous xenon detectors feature a few-percent energy resolution at low energy (figure 9), only surpassed by semiconductor detectors. Relaxing the need to detect primary scintillation would result in the loss of background rejection ability. However, background rejection has been shown not to be a requirement for $\mathrm{CE} \nu \mathrm{NS}$ detection, for sufficiently radioclean detectors such as the CsI[Na] crystal in [2], or the germanium PPCs discussed below (figure 10).

Dedicated studies of the response of gaseous detectors to few-keV nuclear recoils will be necessary to reduce the present uncertainty on parameters such as the quenching factor. Such measurements are planned using a photoneutron source [98] in a 1-kg scale 

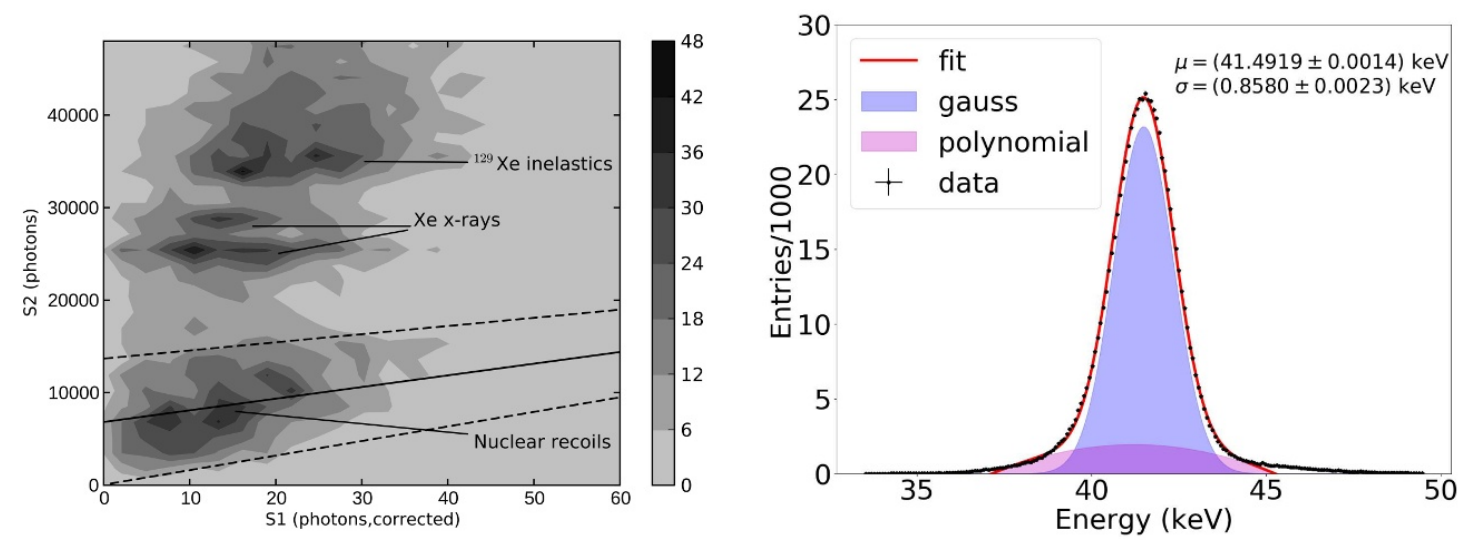

Figure 9. Left: nuclear recoil identification in a gaseous xenon detector by using S1/S2 discrimination [104]. Right: example of energy resolution obtained for $41.5 \mathrm{keV}$ gammas [105]. The very small Fano factor in xenon gas, two orders of magnitude lower than for liquid xenon, allows for excellent energy resolution even in large-scale detectors [106].

NEXT-like detector. In the interim we use a $\mathrm{QF}=20 \%$, similar to that adopted for S2 generation in [104].

One interesting possibility for this detector design is the ability to use different noble gas targets within the same setup. This will allow to compare data taken with xenon, krypton, argon, neon, and even helium. At the time of this writing, a large-volume gaseous detector is already searching for neutrinoless double-beta decay, using high pressure xenon gas: the NEXT-White detector [107]. This device, with internal dimensions of $0.57 \mathrm{~m}$ diameter $\times 0.72 \mathrm{~m}$ length can hold up to $20 \mathrm{kgs}$ of Xe at an operating pressure of 20 bar. Once replaced by the planned upgrade to NEXT-100 in 2020 [106], NEXT-White can be easily adapted to low-energy searches, and used for CE $\nu$ NS studies at the ESS. In the present absence of a dedicated background measurement at shallow depth, we adopt in table 1 a level similar to that for CsI.

\subsection{Low-threshold, multi-kg p-type point contact germanium detectors}

P-type point contact germanium detectors (PPCs, [108]) provide a unique combination of detector mass, ultra-low energy threshold, and background rejection capabilities. As such they have found multiple applications in neutrino (Majorana [109], GERDA [110], TEXONO [111], CONUS [112]) and dark matter searches (CoGeNT [113], CDEX [114]). A continuous improvement in PPC mass and energy threshold [115] has resulted in large devices approaching $0.1 \mathrm{keV}_{e e}$ sensitivity. Inverted coaxial PPCs [116] allow for single multi-kg crystals.

A recently completed $2.95 \mathrm{~kg}$ PPC features a stable $0.18 \mathrm{keV}_{e e}$ threshold, and a 15 ckkd background at threshold (figure 10) during operation in a shallow overburden site at the University of Chicago (6 m.w.e.). This is similar to what can be expected in a ESS basement location (the SNS neutrino alley provides 8 m.w.e.). This background is achieved via a new shielding design that includes a double active veto. Its innermost plastic scintillator layer surrounds the PPC. It is able to tag the neutrino-induced neutron (NIN) background from 


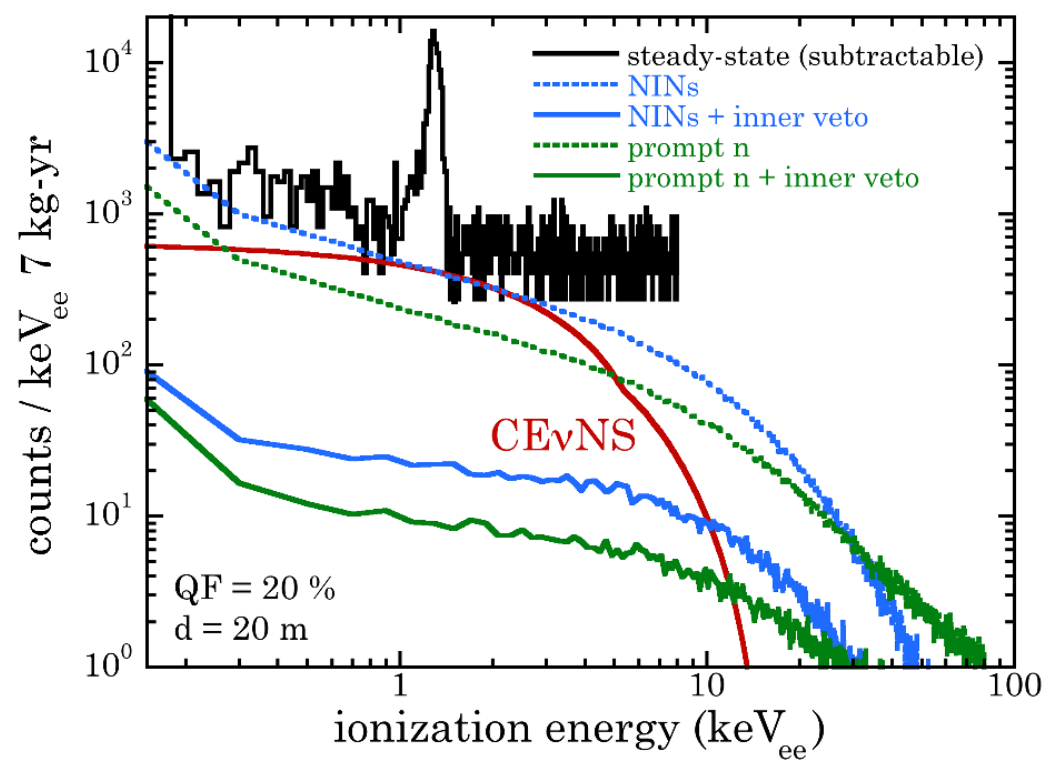

Figure 10. Comparison of expected CE $\nu$ NS signal in Ge PPCs at the ESS to backgrounds. The beam-associated prompt neutron flux and NIN production rate are taken to be $\times 10$ those at the SNS [2], with same spectral hardness, in the simulations shown. The effect of the inner veto uses as input its measured light collection efficiency, and a neutron response for plastic scintillator as in [117]. The subtractable steady-state background achieved at 6 m.w.e. with the PPC described in the text is shown, applying the ESS duty factor for a direct comparison to CE $\nu$ NS. As a reference, the $\mathrm{CE} \nu \mathrm{NS}$ signal to steady-state background ratio for $\mathrm{CsI}[\mathrm{Na}]$ in [2] was a less favorable $\sim 1 / 4$.

lead shielding [2], and beam-related prompt neutrons, with a high efficiency (figure 10). The shield design is intentionally compact at $60 \mathrm{~cm} \times 60 \mathrm{~cm} \times 150 \mathrm{~cm}$. Use of a cryocooler provides unattended operation without access to liquid nitrogen for periods $>1$ year. Special measures were taken in the internal detector design and FPGA-based data-acquisition to obtain an absence of measurable cryocooler-induced microphonic noise. The device is presently unique in its combination of large mass and low energy threshold. However, a further reduction in PPC leakage current is planned, aiming to push the threshold down to $\sim 0.12 \mathrm{keV}_{e e}$. An effort towards the characterization of the sub-keV quenching factor in germanium is also underway [71]. For the purposes of ESS sensitivity calculations, we adopt a germanium mass of $7 \mathrm{~kg}$ (achievable with two PPCs), the already demonstrated background of figure 10, the upgraded threshold, and a quenching factor of $20 \%$ [118] (see table 1).

\subsection{Moderately superheated liquids}

Moderately superheated bubble chambers like those used for the PICO dark matter search [119-121] provide a dramatic insensitivity to electron recoil (ER) backgrounds, the best of any nuclear recoil detector [122]. A recent development in this area are scintillating bubble chambers $[123,124]$. The additional information channel provided by light detection facilitates sub-keV nuclear recoil thresholds, while preserving ER insensitivity. Specifically, a $\sim 0.1 \mathrm{keV}_{\mathrm{nr}}$ threshold is expected from a liquid argon (LAr) bubble chamber. The scin- 

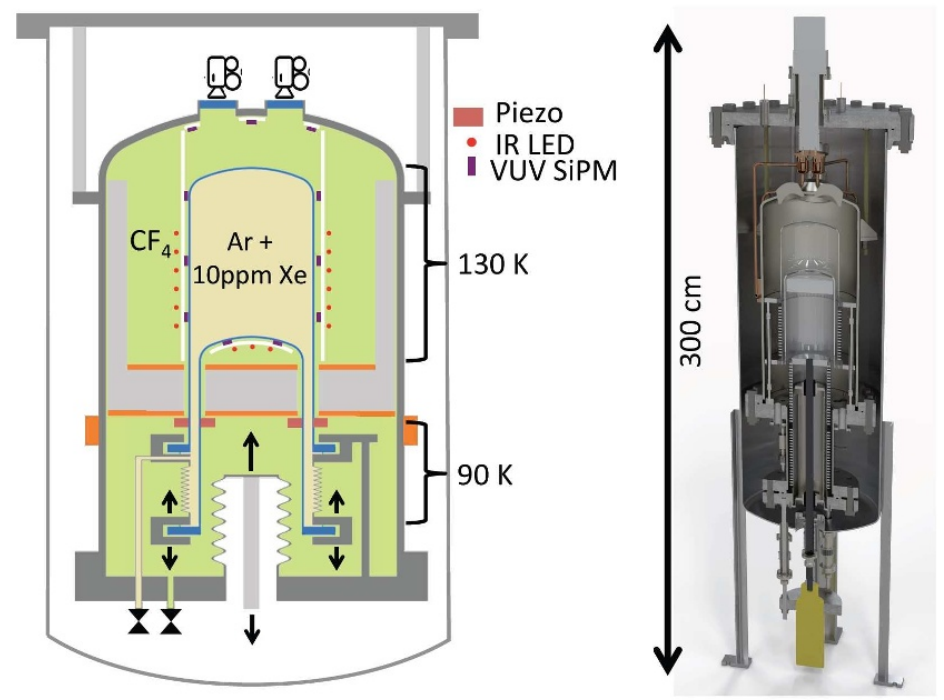

Figure 11. Schematic and model of a 10-kg LAr bubble chamber under construction, showing pressure and temperature control, bubble imaging, and scintillation detection scheme. The design is of a "right side up" type, as in [123] and the upcoming PICO-40 and PICO-500 chambers at SNOLAB. Rendering by Fermilab's PPD/Mechanical Engineering Department.

tillation signal also helps with improving the timing of the detector, otherwise limited to $\sim 25 \mu$ s through the acoustic emission from rapidly expanding bubbles [123, 125].

Besides the mentioned ultra-low energy threshold and insensitivity to ERs, bubble chambers exhibit a few additional features of interest for CE $\nu$ NS detection at the ESS: i) a 3-D event reconstruction of order $1 \mathrm{~mm}$ permits to distinguish neutrino interactions from neutron backgrounds, due to their different spatial distribution. ii) Suitable (but non-scintillating) targets containing light elements such as C, F, and Br exist, and have been tested within the COUPP, PICASSO, and PICO dark matter searches. For these the $\mathrm{CE} \nu \mathrm{NS}$ cross section is small (e.g., $\mathrm{C}_{3} \mathrm{~F}_{8}$, figure 7 ), increasing the difficulty of a measurement. The extreme insensitivity to ERs of order $10^{-9}$ [122] seems crucially important in order to achieve an eventual $\mathrm{CE} \nu \mathrm{NS}$ detection in light targets. iii) A dominant ${ }^{39} \mathrm{Ar} \mathrm{ER}$ background limits the performance of conventional LAr detectors during CE $\nu$ NS measurements $[56,57]$. Use of LAr within a scintillating bubble chamber bypasses this issue, due to the intrinsic insensitivity to beta emitters. iv) The ultra-low $0.1 \mathrm{keV}_{\mathrm{nr}} \mathrm{NR}$ threshold expected from a scintillating LAr bubble chamber makes it highly sensitive to deviations in expected $\mathrm{CE} \nu \mathrm{NS}$ signal rate induced by a finite neutrino magnetic moment (section 4).

PICO experimentation has demonstrated that the thermodynamically-defined threshold for bubble formation is well-defined and predictable [121, 125]. This allows to obtain spectral information by scanning operating parameters (typically pressure), at the expense of an increased exposure. The NR background adopted in table 1 is derived from unshielded runs of a $2 \mathrm{~kg} \mathrm{CF}_{3} \mathrm{I}$ bubble chamber [120] at 6 m.w.e., including the simulated effect of $40 \mathrm{~cm}$ of neutron moderator shielding and $20 \mathrm{~cm}$ of recompression/refrigerant fluid on environmental neutrons at this overburden. 
Presently, two 10-kg scintillating bubble chambers are under construction at Northwestern University and Fermilab (figure 11), one dedicated to dark matter searches, the second intended for $\mathrm{CE} \nu \mathrm{NS}$ experimentation. The time foreseen until their full commissioning is a good match to the ESS start-up schedule.

\section{Physics reach}

As mentioned in section 1, a precision measurement of $\mathrm{CE} \nu \mathrm{NS}$ provides a direct probe to both SM and beyond the standard model (BSM) physics. Paradigmatic examples of the former are the determination of the weak mixing angle at very low momentum transfer [2729], and the study of nuclear structure [17-20]. The program of BSM exploration with $\mathrm{CE} \nu \mathrm{NS}$ is broad (see $[6-15,21-26,30,32-34,126,127]$ for an incomplete list) being most sensitive to a variety of scenarios leading to modified neutrino interactions with nuclei in particular at low momentum transfer - but extending also to the production of new light neutral states, and sterile neutrino searches, among others.

On this front, although part of the rationale for using a variety of targets at the ESS is to demonstrate the $\mathrm{N}^{2}$ dependence of the CE $\nu \mathrm{NS}$ cross-section [55], much more interesting is the synergy in constraining the BSM physics that the use of multiple targets can bring, and the advantages that some of these technologies provide for specific aspects of the phenomenology explored. An additional argument is that of redundancy: any observed deviations from $\mathrm{SM} C \mathrm{C} \nu \mathrm{NS}$ predictions will require independent tests. In this respect of anomaly confirmation, xenon and CsI targets provide a rather unique combination of nearly-identical response to $\mathrm{CE} \nu \mathrm{NS}$ (figure 7), while relying on fundamentally different techniques, subject to systematics not in common.

In what follows we illustrate the potential sensitivity of a $\mathrm{CE} \nu \mathrm{NS}$ experiment at the ESS to SM and BSM physics, for a variety of target nucleus and detection technologies, with three characteristic examples: NSI, the weak mixing angle and neutrino charge radius, and an anomalous neutrino magnetic moment.

\subsection{Parameters and assumptions used in calculations}

For neutrino energies in the $50 \mathrm{MeV}$ range, as it is the case for neutrinos produced from pion DAR, the coherence condition is satisfied for medium-sized nuclei. In the SM the differential cross section for $\mathrm{CE} \nu \mathrm{NS}$ on a nucleus with $Z$ protons and $N$ neutrons reads [3]:

$$
\frac{d \sigma_{S M}\left(T, E_{\nu}\right)}{d T}=\frac{G_{F}^{2}}{2 \pi} \frac{\mathcal{Q}^{2}(Z, N)}{4} F^{2}\left(Q^{2}\right) M\left(2-\frac{M T}{E_{\nu}^{2}}\right)
$$

where $T$ is the recoil energy of the nucleus, $M$ is its mass, $E_{\nu}$ is the incident neutrino energy, $G_{F}$ is the Fermi constant, and $F$ is the form factor of the nucleus evaluated at the squared momentum transfer of the process, $Q^{2}=2 M T$. Here $\mathcal{Q}^{2} \equiv 4\left(Z g_{V, p}+N g_{V, n}\right)^{2}$, with $g_{V, p}=1 / 2-\sin ^{2} \theta_{w}$ and $g_{V, n}=-1 / 2$ being the weak charges of the proton and the neutron, respectively. In our calculations, the weak mixing angle has been set to its value at zero momentum transfer $\sin ^{2} \theta_{w}=0.23867$, following refs. [128, 129]. As for the form factors, most of them are readily available from ref. [130]. For molecules, however, 
since these are not available we take the weighted average between the form factors for the different nuclei. In the case of $\mathrm{C}_{3} \mathrm{~F}_{8}$, in the absence of a form factor for $\mathrm{F}$ we take the corresponding one for $\mathrm{O}$ instead. In the case of CsI we use the Helm form factor parametrization [131], using $s=0.9 \mathrm{fm}$ and $R_{n}=4.83 \mathrm{fm}$.

All sensitivity calculations in this work are obtained in nuclear recoil energy space, after accounting for the effect of the QF on the detector threshold and backgrounds. The differential number of events for the signal reads

$$
\frac{d N}{d T}=\mathcal{N} \sum_{\alpha \equiv \nu_{e}, \nu_{\mu}, \bar{\nu}_{\mu}} \int d E_{\nu} \frac{d \sigma\left(T, E_{\nu}\right)}{d T} \frac{d \phi_{\alpha}\left(E_{\nu}\right)}{d E_{\nu}},
$$

where $\mathcal{N}$ is a normalization constant that depends on the number of protons on target, the neutrino yield per proton, the detection efficiency (or acceptance), the mass of the detector, and its distance to the source. Unless otherwise stated, a common set of assumptions apply to this normalization constant, for all detector configurations considered in this work:

1. the detector distance to the ESS target is set to $20 \mathrm{~m}$;

2. the detector signal acceptance is assumed to be a step function at threshold, with a conservative $80 \%$ acceptance;

3. the running time is restricted to a total of 3 years. We think that this is reasonable, given the much longer running times envisioned for the physics program at the ESS [35]. Increasing it beyond this value may lead to an improvement in our results only if the systematic errors can be reduced with respect to our assumed benchmark values outlined below.

In what respects the expected backgrounds, they can be divided into three classes: $(i)$ steady-state backgrounds (dominated by cosmic ray interactions or by their by-products inside or in the surroundings of a radioclean detector); (ii) beam-related backgrounds, produced by neutrons escaping the target and reaching the detector; and (iii) neutrinoinduced neutrons. While the latter is irreducible, it has been shown that its contribution to the total event rate at the SNS is very small $[2,5]$, and therefore will be neglected here. Beam-related backgrounds have also been neglected (awaiting confirmation from ESS neutron background studies), assuming that similar levels to those in [2] or shown in figure 10 are achievable. Therefore, our main backgrounds are expected to come from the steady-state contribution. For simplicity, this is assumed to follow a uniform distribution in recoil energy.

Table 1 summarizes the detector properties, steady-state background, and QF values assumed for all detectors considered in this work. With the exception of bubble chambers (see below), a Gaussian energy smearing is applied, with a width that depends on the recoil energy as: $\sigma(T)=\sigma_{0} \sqrt{T / T_{\text {thres }}}$, where $\sigma_{0}$ is the energy resolution at the detection threshold $\left(T_{\text {thres }}\right)$, which can be readily extracted from table 1 . For each detector, the recoil energy bin sizes are chosen so that the width of each bin is twice the energy resolution at its center. We consider all the kinematically available range (determined by the condition $\left.T \lesssim 2 E_{\nu}^{2} / M\right)$, for all detector configurations. 


\begin{tabular}{|c|c|c|c|c|c|c|c|c|c|}
\hline Detector Technology & $\begin{array}{c}\text { Target } \\
\text { nucleus }\end{array}$ & $\begin{array}{c}\text { Mass } \\
(\mathrm{kg})\end{array}$ & $\begin{array}{c}\text { Steady-state } \\
\text { background }\end{array}$ & $\begin{array}{c}\mathrm{E}_{t h} \\
\left(\mathrm{keV}_{e e}\right)\end{array}$ & $\begin{array}{c}\mathrm{QF} \\
(\%)\end{array}$ & $\begin{array}{c}\mathrm{E}_{t h} \\
\left(\mathrm{keV}_{\mathrm{nr}}\right)\end{array}$ & $\begin{array}{c}\frac{\Delta E}{E}(\%) \\
\text { at } \mathrm{E}_{t h}\end{array}$ & $\begin{array}{c}\mathrm{E}_{\mathrm{max}} \\
\left(\mathrm{keV}_{\mathrm{nr}}\right)\end{array}$ & $\begin{array}{c}\mathrm{CE} \nu \mathrm{NS} \frac{\mathrm{NR}}{\mathrm{yr}} \\
@ 20 \mathrm{~m},>\mathrm{E}_{t h}\end{array}$ \\
\hline Cryogenic scintillator & $\mathrm{CsI}$ & 22.5 & $10 \mathrm{ckkd}$ & 0.1 & $\sim 10[71]$ & 1 & 30 & 46.1 & 8,405 \\
Charge-coupled device & $\mathrm{Si}$ & 1 & $1 \mathrm{ckkd}$ & 0.007 & $4-30[97]$ & 0.16 & 60 & 212.9 & 80 \\
High-pressure gaseous TPC & $\mathrm{Xe}$ & 20 & $10 \mathrm{ckkd}$ & 0.18 & $20[104]$ & 0.9 & 40 & 45.6 & 7,770 \\
p-type point contact HPGe & $\mathrm{Ge}$ & 7 & $15 \mathrm{ckkd}$ & 0.12 & $20[118]$ & 0.6 & 15 & 78.9 & 1,610 \\
Scintillating bubble chamber & $\mathrm{Ar}$ & 10 & $0.1 \mathrm{c} / \mathrm{kg}$-day & - & - & 0.1 & $\sim 40$ & 150.0 & 1,380 \\
Standard bubble chamber & $\mathrm{C}_{3} \mathrm{~F}_{8}$ & 10 & $0.1 \mathrm{c} / \mathrm{kg}$-day & - & - & 2 & 40 & 329.6 & 515 \\
\hline
\end{tabular}

Table 1. Summary of detector properties, maximum recoil energies considered and expected signal and background rates used in our sensitivity calculations. Backgrounds listed do not include the $4 \times 10^{-2}$ reduction by the ESS duty factor. The germanium QF in [118] will be revisited in an upcoming publication. A rapid dependence of the silicon QF on NR energy [97, 132] is adopted. The concept of QF is ill-defined for bubble chambers (all NR energy is available for nucleation). Their background is integrated above nucleation threshold (in counts per kg and day). Backgrounds for semiconductors are in per $\mathrm{keV}_{e e}$, while for cryogenic CsI and pressurized xenon this is given in per $\mathrm{keV}_{\mathrm{nr}}$, and in both cases are given in counts per $\mathrm{keV}, \mathrm{kg}$ and day (ckkd). We conservatively adopt the background at $\mathrm{E}_{t h}$, which is typically maximal, for all higher energies.

While most detectors under consideration have excellent energy reconstruction capabilities, for bubble chambers only the total event rates are used in the calculations: no bins or energy smearing are used to compute the event rates. For these detectors, however, it is possible to predict and adjust the detection threshold with high precision [121, 125]. Splitting the running time of a bubble chamber into two periods (with different detection thresholds) would lead to an increased sensitivity to NP scenarios which manifest at low recoils but remain unnoticed for events in the higher energy part of the spectrum, as we will see in section 4.4

In order to determine the sensitivity to a NP model characterized by a set of parameters $\{\varepsilon\}$, a binned $\chi^{2}$ is built. Systematic uncertainties are implemented using the pull method, and assumed to be fully correlated among different energy bins while totally independent for signal and background. Thus we introduce two nuisance parameters $\xi_{s i g}$ and $\xi_{b g}$ to parametrize these independent normalization uncertainties. Altogether

$$
\chi^{2}(\{\varepsilon\})=\min _{\xi}\left[\chi^{2}(\{\varepsilon\}, \xi)+\left(\frac{\xi_{\text {sig }}-1}{\sigma_{\text {sig }}}\right)^{2}+\left(\frac{\xi_{b g}-1}{\sigma_{b g}}\right)^{2}\right]
$$

where

$$
\chi^{2}(\{\varepsilon\}, \xi)=\sum_{i} 2\left[N_{i}(\{\varepsilon\}, \xi)-\bar{N}_{i}+\bar{N}_{i} \ln \left(\frac{\bar{N}_{i}}{N_{i}(\{\varepsilon\}, \xi)}\right)\right] .
$$

Here $N_{i}(\{\varepsilon\}, \xi)$ stands for the event rate/s in the $i$-th energy bin (adding up signal and background rates each with its corresponding normalization factor) predicted by the model that is being tested, while $\bar{N}_{i}$ stands for the event rates expected in that bin from the combination of signal and background in the SM. In eq. (4.3), $\sigma_{s i g}$ and $\sigma_{b g}$ are signal and background normalization uncertainties. In the results presented here a $10 \%$ systematic uncertainty has been assumed for the signal prediction. For reference, the corresponding 
value at the COHERENT experiment currently exceeds $20 \%$, after adding all corresponding uncertainties in quadrature. However, the largest contributor to such error is the systematic error on the QF, which according to the results from ref. [71] can be reduced down from a $18 \%$ to approximately a $6 \%$. Therefore, we deem a $10 \%$ representative of the cumulative uncertainties in $\mathrm{QF}$, neutrino flux, nuclear form factor, and signal acceptance by the time data taking starts. While the steady-state background is in principle subject to sizable systematic uncertainties, it will be efficiently measured using beam-off data, as demonstrated at COHERENT $[2,5]$. Therefore, this systematic uncertainty is set to $1 \%$ in our calculations for all detectors under consideration. It is important to stress that, given the large statistics predicted in all considered detectors, most of the sensitivity results presented below are limited by systematics within our simplified treatment and conservative assumptions about their uncertainties. They are, therefore, subject to improvements by dedicated campaigns allowing a better characterization of the systematic uncertanties in each detector.

\subsection{Non-standard neutrino interactions}

From a completely model-independent approach, a useful parametrization of the possible effects of NP at low energies is through the addition of higher-dimensional operators to the SM Lagrangian, that respect the SM gauge group. At $d=5$, the only operator that can be built using just SM fields is the Weinberg operator [133], which coincidentally gives rise to neutrino masses. At $d=6$, the allowed set of operators includes four-fermion operators affecting neutrino production, propagation, and detection processes. For example, operators of the form

$$
2 \sqrt{2} G_{F} \epsilon_{\alpha \beta}^{f f^{\prime}, P}\left(\bar{\nu}_{\alpha} \gamma_{\mu} P_{L} \ell_{\beta}\right)\left(\bar{f}^{\prime} \gamma^{\mu} P f\right)
$$

would induce non-standard charged-current $(\mathrm{CC})$ production and detection processes for neutrinos of flavor $\alpha$, while operators such as

$$
2 \sqrt{2} G_{F} \epsilon_{\alpha \beta}^{f, P}\left(\bar{\nu}_{\alpha} \gamma_{\mu} P_{L} \nu_{\beta}\right)\left(\bar{f} \gamma^{\mu} P f\right)
$$

would lead to flavor-changing neutral-current (NC) interactions of neutrinos with other fermions (if $\alpha \neq \beta$ ), or to a modified $\mathrm{NC}$ interaction rate with respect to the SM expectation (if $\alpha=\beta$ ). In eqs. (4.5) and (4.6), $f$ and $f^{\prime}$ refer to SM fermions, $\ell$ refers to a SM charged lepton and $P$ can be either a left- or a right-handed projection operator $\left(P_{L}\right.$ or $P_{R}$, respectively).

While CC NSI are severely constrained by the study of CC processes, such as meson and muon decays, constraining NC NSI is a much more challenging task. This is so because of the uncertainties involved in computing neutrino-nucleus interactions, and the experimental difficulties in measuring $\mathrm{NC}$ cross sections precisely. In fact, the best constraints available in the literature for these operators come from global fits to oscillation data, which are very sensitive to modifications in the effective matter potential felt by neutrinos as they propagate in a medium [12]. Consequently they can bound vector NSI $\left(\epsilon_{\alpha \beta}^{f, V} \equiv \epsilon_{\alpha \beta}^{f, L}+\epsilon_{\alpha \beta}^{f, R}\right)$ and, since they are due to a totally coherent effect, these bounds extend to NSI induced even by ultra light mediators $\left(M_{\text {med }} \gtrsim 1 / R_{\text {Earth }} \sim \mathcal{O}\left(10^{-12}\right)(\mathrm{eV})\right)$. However, while oscillation 
experiments are sensitive to all flavor-changing NSI, they are only sensitive to differences between the diagonal NSI parameters in flavor space $[12,126]$. This leads to the appearance of new degeneracies involving standard oscillation parameters and NSI operators, such as the so-called generalized mass ordering degeneracy [134-136], with important consequences for the upcoming generation of long-baseline neutrino oscillation experiments.

Conversely $\mathrm{CE} \nu \mathrm{NS}$ experiments at spallation sources allow to constrain two of the three flavor-diagonal coefficients, since the neutrino flux contains both muon and electron neutrinos. In brief, the differential number of signal events in the presence of NC NSI can be obtained from eqs. (4.2) and (4.1) with the effective coupling $\mathcal{Q}^{2}$ in eq. (4.1) modified as [137]:

$$
\begin{aligned}
\mathcal{Q}_{\alpha}^{2(\epsilon)}= & 4\left[Z\left(g_{V, p}+2 \epsilon_{\alpha \alpha}^{u, V}+\epsilon_{\alpha \alpha}^{d, V}\right)+N\left(g_{V, n}+\epsilon_{\alpha \alpha}^{u, V}+2 \epsilon_{\alpha \alpha}^{d, V}\right)\right]^{2} \\
& +4 \sum_{\beta \neq \alpha}\left[Z\left(2 \epsilon_{\alpha \beta}^{u, V}+2 \epsilon_{\alpha \beta}^{d, V}\right)+N\left(\epsilon_{\alpha \beta}^{u, V}+2 \epsilon_{\alpha \beta}^{d, V}\right)\right]^{2},
\end{aligned}
$$

where we explicitly note that its value will now generally depend on the incident neutrino flavor $\alpha$. Notice that both oscillation and $\mathrm{CE} \nu \mathrm{NS}$ experiments are sensitive to the vector NSI.

Thus, the complementarity between neutrino oscillation and coherent scattering data is evident: the addition of coherent scattering data to the global fits from oscillation breaks the degeneracies involving flavor-diagonal NSI, thanks to the direct measurement of the neutrino-nucleus interactions for both electron and muon neutrinos [6, 134]. Furthermore, while in principle incoherent neutrino scattering at higher energies is also sensitive to these operators, when the NSI is induced by light mediators the sensitivity of these scattering experiments (such as $\mathrm{NuTeV}$ [138] or CHARM [139]) becomes limited by the larger minimum momentum transfer required in the inelastic scattering detection [126, 134, 140, 141].

With all these considerations, in what follows, we focus on the determination of the flavor-diagonal NSI coefficients, $\epsilon_{\alpha \alpha}^{f, V}(f=u, d)$, although it should be kept in mind that coherent neutrino scattering is also sensitive to all the off-diagonal NSI operators as well, and competitive bounds should also be expected for those.

Figure 12 shows our results on the expected allowed regions at 90\% CL in the plane $\left(\epsilon_{e e}^{u, V}, \epsilon_{\mu \mu}^{u, V}\right)$ for the six detectors under consideration. In this figure for simplicity, we have assumed that the NSI take place only with up-type quarks; however, similar results are obtained if the NSI are assumed to take place with down-type quarks instead. For illustration we show as well the $90 \%$ CL allowed region from the analysis of the total event rate observed at the COHERENT experiment in ref. [12], following the prescription provided in ref. [2] to perform a fit to NSI using the total event rates. In principle, adding the timing and energy information provided in ref. [142] can help to tighten their constraints to some degree $[15,21]$; however, the final result is subject to uncertainties in the treatment of the background and systematic errors assumed.

The different areas in the two panels in figure 12 correspond to the results obtained with the detector configurations listed in table 1. As seen in the figure, in most cases the shape of the allowed regions is an ellipse in this plane. This can be easily understood as follows. 

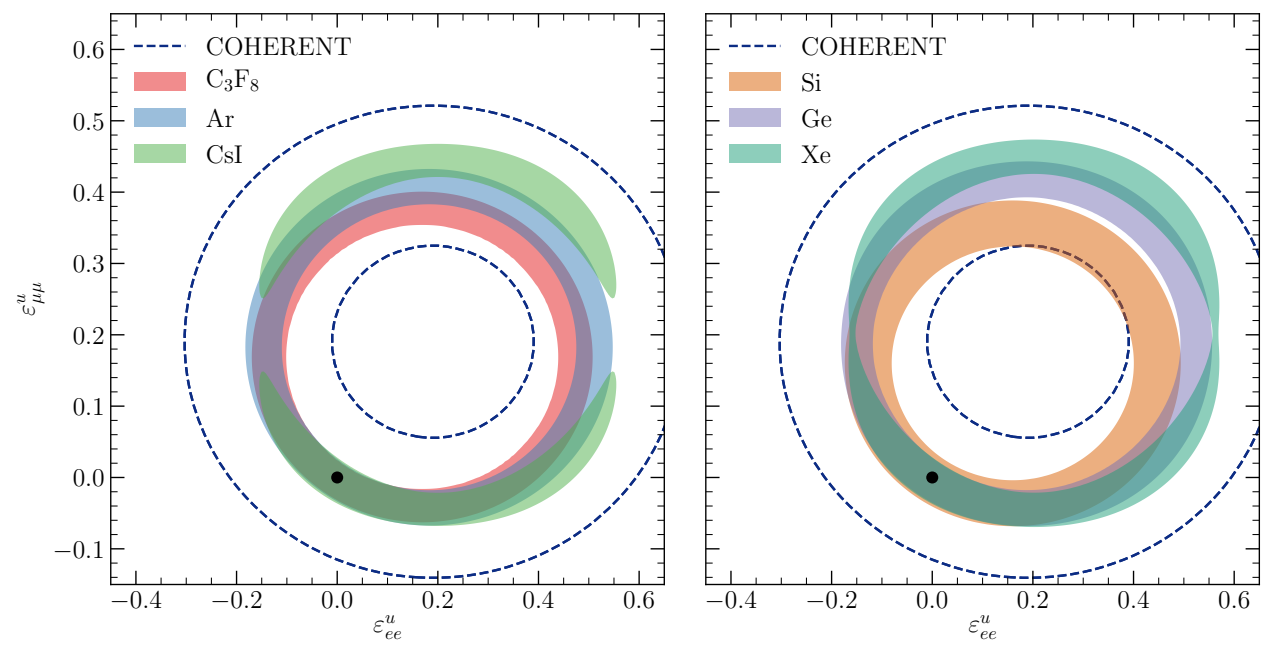

Figure 12. Expected allowed regions in the $\left(\epsilon_{e e}^{u, V}, \epsilon_{\mu \mu}^{u, V}\right)$ plane at the $90 \%$ confidence level (C.L.) for two degrees of freedom (d.o.f.). The different regions correspond to the expected results for the different detectors listed in table 1 , as indicated by the legend. In all cases, the simulated data has been generated for the SM (that is, setting all the operator coefficients to zero), and the results are then fitted assuming NSI. For simplicity, the rest of the NSI parameters not shown in the figure have been assumed to be zero. For comparison, the dashed lines show the allowed regions at $90 \%$ $\mathrm{CL}$ in this plane, as obtained in ref. [6] from an analysis of current data from the COHERENT experiment [2], see text for details.

From eqs. (4.2), (4.1), and (4.7) one can trivially compute the expected total number of events (adding up the contributions from the three components of the beam) in each bin as a function of the two NSI coefficients in each. Requiring that the NSI-induced correction is of the same relative size in all bins and that the total number of events is compatible with the SM expectation, it is straightforward to show that the allowed confidence regions in the plane $\epsilon_{e e}^{u}-\epsilon_{\mu \mu}^{u}$ obey the equation of an ellipse:

$$
\left[R+\epsilon_{e e}^{u, V}\right]^{2}+2\left[R+\epsilon_{\mu \mu}^{u, V}\right]^{2}=3 R^{2}
$$

where $R \equiv \frac{Z g_{V, p}+N g_{V, n}}{2 Z+N}$ only depends on the target nucleus and the SM weak couplings to protons and neutrons. In the SM, given that $g_{V, p} \ll g_{V, n}$ this constant can be safely approximated to $R \simeq g_{V, n} /(2 r+1)$, where $r \equiv Z / N$ is the ratio of protons to neutrons in the nucleus. From eq. (4.8) it follows that the shape of the allowed confidence regions in this plane will be very similar for different target nuclei as long as they have a similar value of $r$. For reference table 2 summarizes the values of $Z, N, r$, and the nuclear masses assumed for different nuclei.

As seen in figure 12, the allowed regions are in good agreement with eq. (4.8), for most of the detectors under consideration. However, from the figure we also see that for some detectors, in particular for the CsI target (and also in part for Xe target), the degeneracy in the allowed region in the $\left(\epsilon_{e e}^{u, V}, \epsilon_{\mu \mu}^{u, V}\right)$ plane implied by eq. (4.8) is partly broken. This is so because eq. (4.8) has been obtained under the approximation of a constant - flavourand energy-independent — shift of the event rates in all bins. Clearly the degeneracy 


\begin{tabular}{|c|cccc|}
\hline Nucleus & $Z$ & $N$ & $r$ & $M$ (a.m.u.) \\
\hline${ }^{132} \mathrm{Xe}$ & 54 & 78 & 0.69 & 131.29 \\
${ }^{40} \mathrm{Ar}$ & 18 & 22 & 0.81 & 39.95 \\
${ }^{72} \mathrm{Ge}$ & 32 & 40 & 0.8 & 75.92 \\
${ }^{28} \mathrm{Si}$ & 14 & 14 & 1.0 & 27.98 \\
${ }^{12} \mathrm{C}$ & 6 & 6 & 1.0 & 12.01 \\
${ }^{19} \mathrm{~F}$ & 9 & 10 & 0.9 & 19.00 \\
${ }^{133} \mathrm{Cs}$ & 55 & 78 & 0.71 & 132.91 \\
${ }^{127} \mathrm{I}$ & 53 & 74 & 0.72 & 126.90 \\
${ }^{20} \mathrm{Ne}$ & 10 & 10 & 1.0 & 20.18 \\
\hline
\end{tabular}

Table 2. Main properties of the nuclei for the different target nuclei considered in this work. The different columns indicate the isotope considered, together with the number of protons and neutrons, the ratio between them $r$, and the value of the nuclear mass in atomic mass units (a.m.u.). For the detectors using $\mathrm{CsI}$ and $\mathrm{C}_{3} \mathrm{~F}_{8}$ we take the weighted average between the two elements in the molecule.

will be broken if somehow the experiment is capable of discriminating between muon and electron neutrino flavors at some level. A possibility to do this, is through the addition of timing information, which allows to distinguish between the prompt component of the beam (which contains just $\nu_{\mu}$ ) and the delayed component (which contains a mixture of $\nu_{e}$ and $\bar{\nu}_{\mu}$ ). Unfortunately, due to the very long proton pulses this would not be possible at the ESS source.

One must notice, however, that the prompt signal is also characterized by a lower neutrino energy $\left(E_{\nu_{\mu}} \sim 30 \mathrm{MeV}\right)$. Therefore, it should be possible to distinguish its contribution using a detector with good energy resolution that allows to observe not only the bulk of events at low energies (which receives equal contributions from the three components of the beam) but also the tail at high recoil energies, above the maximum recoil allowed for the prompt signal. For large enough statistics, this would allow to obtain partial flavor discrimination, by comparing the event rates below and the maximum recoil allowed for the prompt flux. For illustration, we show in figure 13 the expected event rates, where the contribution per flavor is shown separately. As shown in this figure, above the maximum recoil energy allowed for the prompt component the event rates are given almost exclusively by $\nu_{e}$ and $\bar{\nu}_{\mu}$ scattering (albeit with a small contribution from $\nu_{\mu}$ in the first bin, due to smearing by the energy resolution). Consequently in the case of detectors with high statistics, good energy resolution and no saturation, the ellipse is broken in this plane. This is the case for the CsI and Xe detectors, as seen in figure 12 .

Furthermore, from eq. (4.8) it is clear that an alternative form of breaking this degeneracy is through the combination of data obtained using different target nuclei, as long as they have different values of $r$. This is true even if only information on the total event rates is available (without any time nor energy information). While the combination can be done using different detectors among the possibilities listed in table 1, a more convenient option is available in the case of the gas TPC, since the detector can operate not only with 


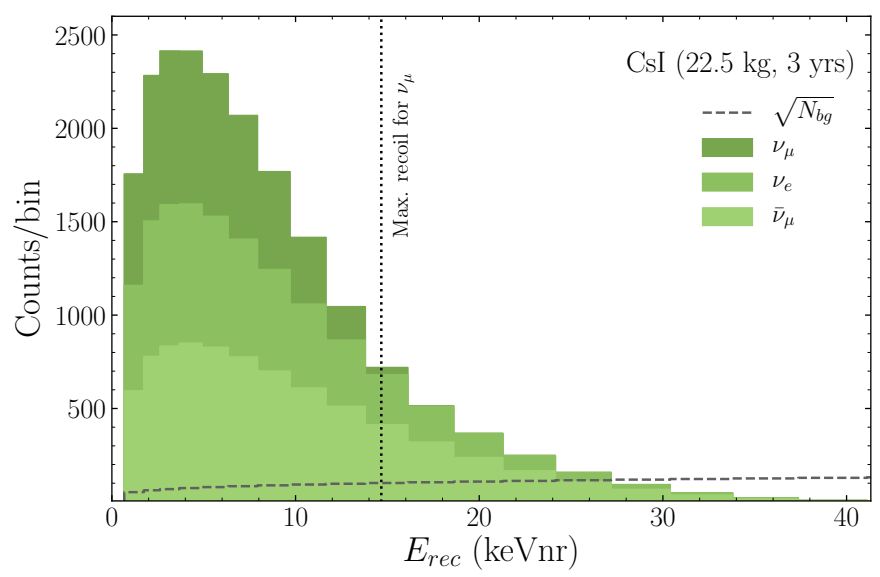

Figure 13. Expected event rates per bin in nuclear recoil energy, for the CsI detector. The contributions from the scattering of the different beam components are shown separately by the shaded histograms, as indicated by the legend. For comparison, the square root of the number of background events in each bin is also shown by the dashed histogram lines. The vertical dotted line indicates the maximum recoil energy allowed by a neutrino with energy $E_{\nu}=29.8 \mathrm{MeV}$, that is, the energy of the monochromatic prompt $\nu_{\mu}$ flux.

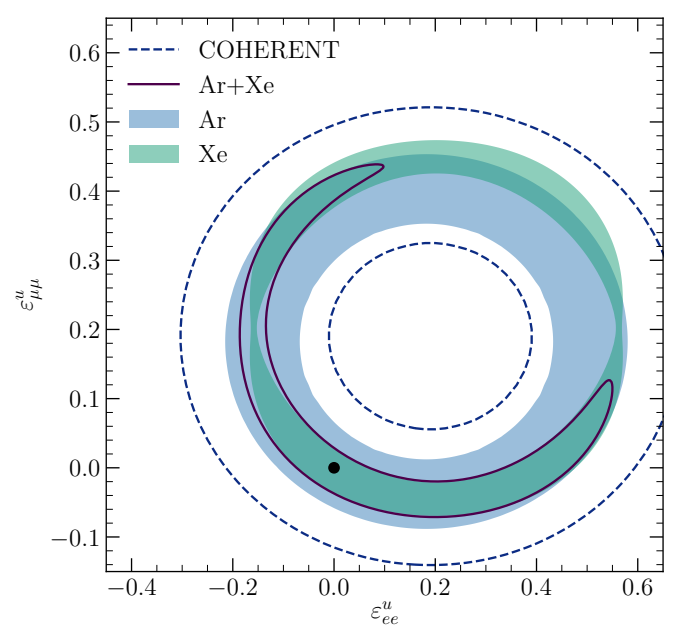

Figure 14. Expected allowed regions in the $\left(\epsilon_{e e}^{u, V}, \epsilon_{\mu \mu}^{u, V}\right)$ plane at the $90 \%$ C.L. for 2 d.o.f, for the gas TPC detector operating with two different nuclei (separate runs, each of them for 3 years), as well as for a configuration where the detector is filled with each of the two gases during half of the total data taking period (1.5 years running with ${ }^{132} \mathrm{Xe}, 1.5$ years with ${ }^{40} \mathrm{Ar}$ ). In all cases, the simulated data has been generated for the SM and the results are then fitted assuming NSI. For simplicity, the values for the rest of NSI parameters not shown in this figure have been set to zero.

xenon but with other noble gases as well (Ne, He, or Ar for instance). As illustration of this possibility, we show figure 14 the expected sensitivity regions in this plane using the gas TPC detector. In this case we show three different results: $(i)$ the expected regions obtained using Xe as the target nucleus; (ii) the expected regions using Ar instead; and (iii) the results obtained using a combined run, where the detector uses Xe during the 


\begin{tabular}{|c|ccccccc|c|}
\hline & $\mathrm{Ar}$ & $\mathrm{C}_{3} \mathrm{~F}_{8}$ & $\mathrm{CsI}$ & $\mathrm{Ge}$ & $\mathrm{Si}$ & $\mathrm{Xe}$ & $\mathrm{Xe}+\mathrm{Ar}$ & $\mathrm{COH}-\mathrm{SNS}$ \\
\hline $\sin ^{2} \theta_{W}$ & $0.239_{-0.022}^{+0.028}$ & $0.239_{-0.020}^{+0.025}$ & $0.239_{-0.026}^{+0.032}$ & $0.239_{-0.024}^{+0.029}$ & $0.239_{-0.029}^{+0.032}$ & $0.239_{-0.026}^{+0.033}$ & $0.239_{-0.029}^{+0.020}$ & $0.248 \pm 0.094[127]$ \\
\hline$\left\langle r_{e e}^{2}\right\rangle$ & {$[-65,20]$} & {$[-58,18]$} & {$[-67,16]$} & {$[-67,20]$} & {$[-54,18]$} & {$[-70,17]$} & {$[-55,20]$} & {$[-65,6][21]$} \\
$\left\langle r_{\mu \mu}^{2}\right\rangle$ & {$[-51,7]$} & {$[-46,6]$} & $-[59,7]$ & {$[-54,7]$} & {$[-43,6.5]$} & {$[-60,7.5]$} & {$[-28,7]$} & {$[-60,10][21]$} \\
$\left|\left\langle r_{e \mu}^{2}\right\rangle\right|$ & $<15$ & $<12$ & $<21$ & $<17$ & $<11$ & $<21$ & $<17$ & $<35[21]$ \\
\hline$\mu_{\nu_{\mu}}$ & $<9$ & $<11$ & $<9$ & $<7$ & $<6$ & $<9$ & $<10$ & $<31[21]$ \\
\hline
\end{tabular}

Table 3. Allowed ranges at $90 \%$ C.L. for the weak mixing angle (given as best fit $\pm 1.64 \sigma$ ), neutrino charge radii for three flavour projections (in units of $10^{-32} \mathrm{~cm}^{2}$, and after marginalizing over the other two flavour projections), and the $\nu_{\mu}$ magnetic moment (90\% CL upper bound in units of $\left.10^{-10} \mu_{\mathrm{B}}\right)$.

first half of the data taking period and Ar during the other half. From the figure we see how the combination of runs with the two selected nuclei leads to a substantially improved sensitivity.

\subsection{Weak mixing angle and neutrino charge radii}

The weak mixing angle is a fundamental parameter in the SM. While its value has been precisely measured at high energies in collider experiments, its determination at low energy is a challenging task from the experimental point of view. At low energies, it can be determined from measurements of parity violation in Cs atoms [143, 144], the parity-violating asymmetry in Moller scattering [145], deep inelastic scattering of polarized electrons in deuteron [146], and neutrino scattering on nuclei [138]. While most of these measurements seem to agree well within error bars, the $\mathrm{NuTeV}$ result [138] shows a tension at the $3 \sigma$ CL.

As seen in eq. (4.1), the weak mixing angle enters the neutrino-nucleus coherent scattering cross section through the value of $g_{V, p}$. Therefore, its effect on the number of events is going to be much more subleading than for NSI, and its impact on the observable number of events will be a change in the normalization of the event sample which, in this case, will be flavor-universal. However, while the weak mixing angle affects the coupling to protons it does not affect the coupling to neutrons and, therefore, an enhanced sensitivity is expected also in this case by combining results obtained for nuclei with different proton-to-neutron ratios $r$.

Our results on the expected sensitivity for this parameter are shown in table 3 for different detectors under consideration. For reference, the current bounds derived in ref. [127] from current COHERENT results, are also included. As seen in the table, any of the experiments considered here can lead to an improvement on the determination of the weak mixing angle in $\mathrm{CE} \nu \mathrm{NS}$ by a factor $\mathcal{O}(3)$. Notice also that the sensitivity of all dectors to this parameter is comparable because it is mostly limited by the assumed $10 \%$ normalization uncertainty.

The determination of the weak mixing angle is tightly related to the sensitivity to the effective neutrino charge radius, $\left\langle r_{\nu}^{2}\right\rangle$, defined as [147]

$$
\left\langle r_{\nu}^{2}\right\rangle=\left.6 \frac{d F_{\nu}\left(q^{2}\right)}{d q^{2}}\right|_{q} ^{2}=0,
$$


where $F_{\nu}$ is the electromagnetic form factor of the neutrino. The inclusion of this form factor affects the scattering of neutrinos with other charged particles in the SM, and effectively induces a shift in the value of the effective mixing angle [148-150],

$$
\sin ^{2} 2 \theta_{w} \rightarrow \sin ^{2} 2 \theta_{w}\left(1+\frac{1}{3} m_{W}^{2}\left\langle r_{\nu}^{2}\right\rangle\right)
$$

where $m_{W}$ is the mass of the $W$ boson.

The SM prediction for the neutrino charge radii gives a value that depends on the neutrino flavor $\alpha \equiv e, \mu, \tau$, as [151-153]:

$$
\left\langle r_{\nu_{\alpha}}^{2}\right\rangle=\frac{-G_{F}}{2 \sqrt{2} \pi^{2}}\left[3-2 \ln \left(\frac{m_{\ell_{\alpha}}^{2}}{m_{W}^{2}}\right)\right],
$$

where $m_{\ell_{\alpha}}$ is the mass of the charged lepton of the same flavor $\alpha$. Therefore, determining the weak mixing angle precisely allows to obtain an upper limit for the value of the charge radius of each neutrino flavor state. Numerically, however, these lie in the range $[-0.83,-0.3] \times$ $10^{-32} \mathrm{~cm}^{2}$ and are therefore very challenging to observe.

However, in BSM scenarios the charge radii may receive additional contributions and, therefore, a measurement of their value well above the SM expectation would be a clear signal of NP. Moreover in BSM models with neutrino masses, due to the mismatch between the mass and flavor bases, transition charge radii $\left\langle r_{\alpha \beta}^{2}\right\rangle$ may be generated for the flavor states. In the most general case where both diagonal and transition charge radii are considered, the effective weak coupling is modified as [21, 150]:

$$
\mathcal{Q}_{\alpha}^{2(r)}=4\left[Z\left(g_{V, p}-Q_{\alpha \alpha}\right)+N g_{V, n}\right]^{2}+4 Z^{2} \sum_{\beta \neq \alpha}\left|Q_{\alpha \beta}\right|^{2}
$$

where $Q_{\alpha \beta}$ is defined as

$$
Q_{\alpha \beta} \equiv \frac{2}{3} m_{W}^{2} \sin ^{2} \theta_{w}\left\langle r_{\alpha \beta}^{2}\right\rangle .
$$

Note that, unlike for NSI, in this case the modification to the neutrino cross section will be proportional to the number of protons in the nucleus and therefore the expected effect on the number of events will be different. Moreover, in presence of transition charge radii additional modifications to the SM cross section, proportional to $Z^{2}$, are also expected. Finally in this scenario we also expect, as in the case of the weak mixing angle, an improvement in sensitivity through the combination of different target nuclei with different values of $r$. This will greatly help to cancel the effect of systematic uncertainties and to increase the sensitivity to the values of $Q_{\alpha \beta}$.

Our results for the determination of the neutrino charge radii are summarized in table 3 , for the different detectors under consideration. For reference, the current bounds derived from COHERENT data in ref. [21], are also included. As seen in the table, for any of the detectors considered, an improvement with respect to the present results from COHERENT is expected regarding the sensitivity to the charge radius for $\nu_{\mu}$ (although they cannot reach the SM expectation), as well as the flavour transition charge radii. 


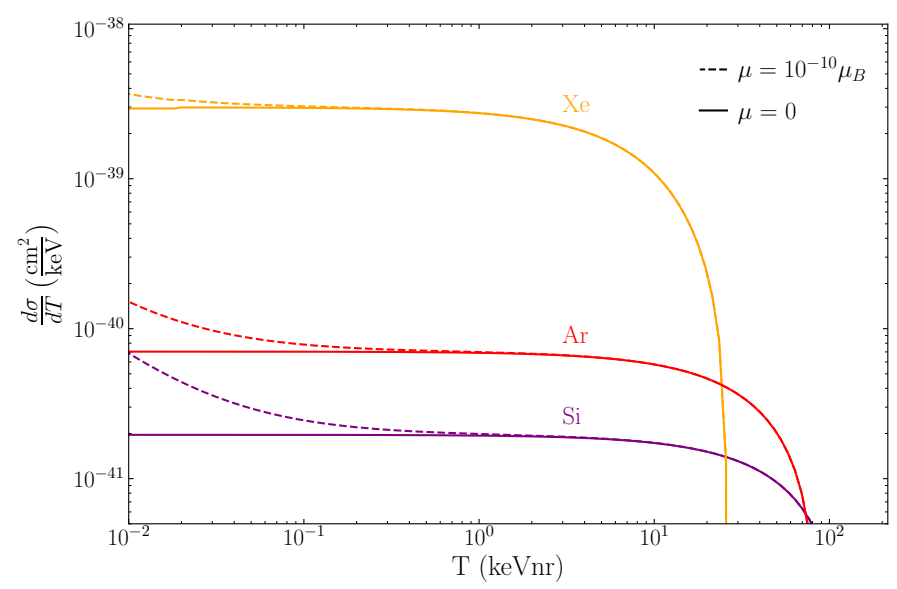

Figure 15. Differential cross section for $\mathrm{CE} \nu \mathrm{NS}$, as a function of the recoiling energy of the nucleus, in the SM and in presence of a non-vanishing neutrino magnetic moment $\mu=10^{-10} \mu_{B}$. The different colors correspond to different target nuclei, as indicated by the labels. The incident neutrino energy is set to $E_{\nu}=40 \mathrm{MeV}$ in all cases, and the cross section in the $\mathrm{SM}(\mu=0)$ is also shown for comparison.

\subsection{Neutrino magnetic moment}

In presence of a neutrino magnetic moment, the SM cross section receives an extra contribution coming from the exchange of a photon with the nucleus. The differential cross section reads [149]:

$$
\frac{d \sigma}{d T}=\frac{d \sigma_{S M}}{d T}+\frac{\pi \alpha^{2}}{m_{e}^{2}}\left[\frac{1}{T}-\frac{1}{E_{\nu}}+\frac{T}{4 E_{\nu}^{2}}\right] Z^{2} F_{\mathrm{em}}^{2}\left(Q^{2}\right)\left(\frac{\mu}{\mu_{B}}\right)^{2},
$$

where $m_{e}$ is the electron mass, $\mu_{B}$ is the Bohr's magneton, and $\alpha$ is the electromagnetic fine constant. It should be noted that, since the effect due to a non-zero magnetic moment is only relevant for very low recoils, the electromagnetic form factor $F_{\mathrm{em}}\left(Q^{2}\right)$ can be safely approximated to one.

Figure 15 shows the differential cross section in eq. (4.14) as a function of the recoil energy, for an incident neutrino with energy $E_{\nu}=40 \mathrm{MeV}$. The different lines correspond to different target nuclei, as indicated in the legend. As can be seen from the figure, the effect of a finite neutrino magnetic moment on event rates is noticeable for recoil energies below 0.5-1 $\mathrm{keV}_{\mathrm{nr}}$, depending on the target nucleus being considered. Therefore, it is expected that detectors with the lowest possible recoil energy threshold, like those showcased in this paper, will be most sensitive to this neutrino property. In this respect, the scintillating bubble chamber and the charged coupled device stand out among the detectors listed in table 1, with lower thresholds well below those for the rest of technologies considered.

Our results of the expected sensitivity to neutrino magnetic moments with the detectors under consideration are summarized in table 3. For the Ar bubble chamber, in spite of having the lowest detection threshold, the lack of energy resolution makes this configuration systematics-limited. However, bubble chamber detectors offer the possibility of adjusting their low energy threshold. While the event rates decrease as the detector threshold is 
increased (see figure 7), splitting the running time of a bubble chamber into two periods (with different detection thresholds) would lead to an efficient cancellation of systematic uncertainties, and to a boost in the sensitivity to this model. Therefore, the results shown in table 3 for this detector are obtained for a configuration where the data taking time is split evenly between two exposures with a different detector threshold: 1.5 years running with $E_{t h}=0.1 \mathrm{keVnr}$, and 1.5 years running with $E_{t h}=1 \mathrm{keVnr}$. In this case, the systematic uncertainties are taken to be fully correlated between the two samples. To this end, the threshold can be trivially alternated between consecutive events, by varying the operating pressure of the chamber.

Neutrino magnetic moments arise in a variety of models of NP and, in particular, they do not need to be flavor-universal. Therefore, being as general as possible, we allow different magnetic moments for the different neutrino flavors. However, reactor and solar experiments (among others) bound the $\nu_{e}$ magnetic moments at the level of $10^{-11} \mu_{\mathrm{B}}[128,154]$ and render its effect well beyond the reach of any of the $\mathrm{CE} \nu \mathrm{NS}$ experiments considered here. For this reason we only show on table 3 our results on the sensitivity to $\mu_{\nu_{\mu}}$ for which current scattering experiments yield a bound of $6.8 \times 10^{-10}$ at $90 \%$ CL [21] which, as seen in the table, can be improved with some of the considered setups.

\section{Conclusions}

Operating at a proton energy of $2 \mathrm{GeV}$ and $5 \mathrm{MW}$ power, the ESS will soon become not just the most intense source of spallation neutrons, but also of pion DAR neutrinos. Its predicted neutrino yield is unmatched by present or planned spallation sources. As such, it provides an extensive enhancement in sensitivity to many areas of phenomenology reachable via $\mathrm{CE} \nu \mathrm{NS}$ experimentation. While we have not included this possibility in our analysis, it should be noted that presently contemplated future ESS upgrades include doubling the beam spill rate to $28 \mathrm{~Hz}$, and/or increasing the proton energy to $2.5-3 \mathrm{GeV}$. Both options would lead to further improvements in sensitivity.

Recent work [71] has shown that systematic uncertainties affecting CE $\nu$ NS experiments can be reduced to a cumulative $\sim 10 \%$ level. Realistically, and from a present-day perspective, further progress in this direction should be considered very hard to achieve. In this context, the subdominant statistical uncertainty possible for $\mathrm{CE} \nu \mathrm{NS}$ measurements at the ESS acquires a special relevance: the experimentation we have described will provide close to the best possible sensitivity to neutrino properties that can be expected from $\mathrm{CE} \nu \mathrm{NS}$ studies at spallation sources.

In this work we have explored the sensitivity to a few representative NP scenarios which illustrate the potential of our proposal from a quantitative perspective: NSI, precise determination of the weak mixing angle, neutrino charge radii and neutrino magnetic moments. We find that, while flavour discrimination associated to temporal separation of the signal would not be possible at the ESS, the large statistics expected still allows for a partial separation between different neutrino flavors using the energy information. On the detector side, we have considered a suite of innovative detector technologies that originate in the fields of dark matter detection and double-beta decay searches (listed in table 1), 
and that will allow to maximally profit from the order-of-magnitude increase in neutrino flux. Specifically, we find that any of the detectors considered here can lead to an improved precision of the determination of the weak mixing angle in $\mathrm{CE} \nu \mathrm{NS}$ by a factor $\mathcal{O}(3)$ and to tighter bounds on $\nu_{\mu}$ magnetic moment by a similar factor. A substantial enhancement on the sensitivity to NSI is also expected as shown in figure 12. We have also explored the improvement in reach obtained from a combination of nuclear targets and quantified this effect in the context of NSI, see figure 14 .

Finally, we want to emphasize that our proposal is not premature, as the time to develop and implement these innovative nuclear recoil detector technologies is a good match to the start of the ESS users' program in 2023. By employing a variety of detectors, we expect to provide an enhanced sensitivity to neutrino properties, and the ability to confirm or refute any deviations from the SM that might be observed via $\mathrm{CE} \nu \mathrm{NS}$ at the ESS.

\section{Acknowledgments}

We are indebted to Z. Lazic, R. Linander, M. Lindroos, and V. Santoro for their input regarding ESS specifics. We also thank O. Borshchev at Luminnotech for kindly providing us with NOL wavelength shifter samples, and M. McClish and P. Waer at RMD Inc. for LAAPD-related consultations.PC warmly thanks B. Dutta for useful discussions. IE acknowledges support from the FPU program fellowship FPU15/0369. PC thanks the CERN Theory division for support and hospitality during the final stages of this work.

MCGG thanks the Department of Physics at Columbia University for their hospitality. This work was supported by the MINECO grants FPA2017-85985-P and FPA2016-76005C2-1-P, by PROMETEO/2019/083, by USA-NSF grants PHY-1620628, PHY-1806722, and PHY-1812702, USA-DARPA award W911NF1810222, by EU Networks FP10 ITN ELUSIVES (H2020-MSCA-ITN-2015-674896) and INVISIBLES-PLUS (H2020-MSCA-RISE2015-690575), and by AGAUR (Generalitat de Catalunya) grant 2017-SGR-929. This work was supported in part by the Kavli Institute for Cosmological Physics at the University of Chicago through an endowment from the Kavli Foundation and its founder Fred Kavli.

Open Access. This article is distributed under the terms of the Creative Commons Attribution License (CC-BY 4.0), which permits any use, distribution and reproduction in any medium, provided the original author(s) and source are credited.

\section{References}

[1] A. Drukier and L. Stodolsky, Principles and applications of a neutral current detector for neutrino physics and astronomy, Phys. Rev. D 30 (1984) 2295 [INSPIRE].

[2] COHERENT collaboration, Observation of coherent elastic neutrino-nucleus scattering, Science 357 (2017) 1123 [arXiv: 1708.01294] [INSPIRE].

[3] D.Z. Freedman, Coherent neutrino nucleus scattering as a probe of the weak neutral current, Phys. Rev. D 9 (1974) 1389 [INSPIRE]. 
[4] J.I. Collar et al., Coherent neutrino-nucleus scattering detection with a CsI[Na] scintillator at the SNS Spallation Source, Nucl. Instrum. Meth. A 773 (2015) 56 [arXiv:1407.7524] [INSPIRE].

[5] B.J. Scholz, First observation of coherent elastic neutrino-nucleus scattering, Ph.D. thesis, Chicago University, Cham, U.S.A. (2017), arXiv:1904.01155 [INSPIRE].

[6] P. Coloma, M.C. Gonzalez-Garcia, M. Maltoni and T. Schwetz, Coherent enlightenment of the neutrino dark side, Phys. Rev. D 96 (2017) 115007 [arXiv: 1708. 02899] [INSPIRE].

[7] J.B. Dent et al., Probing light mediators at ultralow threshold energies with coherent elastic neutrino-nucleus scattering, Phys. Rev. D 96 (2017) 095007 [arXiv:1612.06350] [INSPIRE].

[8] J. Liao and D. Marfatia, COHERENT constraints on nonstandard neutrino interactions, Phys. Lett. B 775 (2017) 54 [arXiv:1708.04255] [INSPIRE].

[9] J.B. Dent et al., Accelerator and reactor complementarity in coherent neutrino-nucleus scattering, Phys. Rev. D 97 (2018) 035009 [arXiv:1711.03521] [INSPIRE].

[10] Y. Farzan, M. Lindner, W. Rodejohann and X.-J. Xu, Probing neutrino coupling to a light scalar with coherent neutrino scattering, JHEP 05 (2018) 066 [arXiv:1802.05171] [INSPIRE].

[11] M. Abdullah et al., Coherent elastic neutrino nucleus scattering as a probe of a $Z^{\prime}$ through kinetic and mass mixing effects, Phys. Rev. D 98 (2018) 015005 [arXiv:1803.01224] [INSPIRE].

[12] I. Esteban et al., Updated constraints on non-standard interactions from global analysis of oscillation data, JHEP 08 (2018) 180 [arXiv:1805.04530] [INSPIRE].

[13] D. Aristizabal Sierra, V. De Romeri and N. Rojas, COHERENT analysis of neutrino generalized interactions, Phys. Rev. D 98 (2018) 075018 [arXiv: 1806.07424] [INSPIRE].

[14] I.M. Shoemaker, COHERENT search strategy for beyond standard model neutrino interactions, Phys. Rev. D 95 (2017) 115028 [arXiv:1703.05774] [INSPIRE].

[15] C. Giunti, General COHERENT constraints on neutrino non-standard interactions, arXiv: 1909.00466 [INSPIRE].

[16] P.B. Denton, Y. Farzan and I.M. Shoemaker, Testing large non-standard neutrino interactions with arbitrary mediator mass after COHERENT data, JHEP 07 (2018) 037 [arXiv: 1804.03660] [INSPIRE].

[17] M. Cadeddu, C. Giunti, Y.F. Li and Y.Y. Zhang, Average CsI neutron density distribution from COHERENT data, Phys. Rev. Lett. 120 (2018) 072501 [arXiv:1710. 02730] [inSPIRE].

[18] E. Ciuffoli, J. Evslin, Q. Fu and J. Tang, Extracting nuclear form factors with coherent neutrino scattering, Phys. Rev. D 97 (2018) 113003 [arXiv:1801.02166] [INSPIRE].

[19] E. Ciuffoli, J. Evslin, Q. Fu and J. Tang, Extracting nuclear form factors with coherent neutrino scattering, Phys. Rev. D 97 (2018) 113003 [arXiv:1801.02166] [INSPIRE].

[20] D.K. Papoulias et al., Constraining nuclear physics parameters with current and future COHERENT data, Phys. Lett. B 800 (2020) 135133 [arXiv:1903.03722] [INSPIRE].

[21] M. Cadeddu et al., Neutrino, electroweak and nuclear physics from COHERENT elastic neutrino-nucleus scattering with a new quenching factor, arXiv:1908.06045 [INSPIRE].

[22] D.K. Papoulias and T.S. Kosmas, COHERENT constraints to conventional and exotic neutrino physics, Phys. Rev. D 97 (2018) 033003 [arXiv:1711.09773] [INSPIRE]. 
[23] J. Billard, J. Johnston and B.J. Kavanagh, Prospects for exploring new physics in coherent elastic neutrino-nucleus scattering, JCAP 11 (2018) 016 [arXiv:1805.01798] [INSPIRE].

[24] M. Cadeddu et al., Neutrino charge radii from COHERENT elastic neutrino-nucleus scattering, Phys. Rev. D 98 (2018) 113010 [arXiv:1810.05606] [INSPIRE].

[25] O.G. Miranda, D.K. Papoulias, M. Tórtola and J.W.F. Valle, Probing neutrino transition magnetic moments with coherent elastic neutrino-nucleus scattering, JHEP 07 (2019) 103 [arXiv: 1905.03750] [INSPIRE].

[26] D.K. Papoulias, COHERENT constraints after the Chicago-3 quenching factor measurement, arXiv:1907.11644 [INSPIRE].

[27] B.C. Cañas, E.A. Garcés, O.G. Miranda and A. Parada, Future perspectives for a weak mixing angle measurement in coherent elastic neutrino nucleus scattering experiments, Phys. Lett. B 784 (2018) 159 [arXiv:1806. 01310] [INSPIRE].

[28] M. Cadeddu and F. Dordei, Reinterpreting the weak mixing angle from atomic parity violation in view of the Cs neutron rms radius measurement from COHERENT, Phys. Rev. D 99 (2019) 033010 [arXiv: 1808.10202] [INSPIRE].

[29] X.-R. Huang and L.-W. Chen, Neutron skin in CsI and low-energy effective weak mixing angle from COHERENT data, Phys. Rev. D 100 (2019) 071301 [arXiv:1902.07625] [INSPIRE].

[30] T.S. Kosmas, D.K. Papoulias, M. Tortola and J.W.F. Valle, Probing light sterile neutrino signatures at reactor and Spallation Neutron Source neutrino experiments, Phys. Rev. D 96 (2017) 063013 [arXiv: 1703.00054] [inSPIRE].

[31] C. Blanco, D. Hooper and P. Machado, Constraining sterile neutrino interpretations of the LSND and MiniBooNE anomalies with coherent neutrino scattering experiments, arXiv: 1901.08094 [INSPIRE].

[32] S.-F. Ge and I.M. Shoemaker, Constraining photon portal dark matter with Texono and COHERENT data, JHEP 11 (2018) 066 [arXiv:1710.10889] [INSPIRE].

[33] V. Brdar, W. Rodejohann and X.-J. Xu, Producing a new fermion in coherent elastic neutrino-nucleus scattering: from neutrino mass to dark matter, JHEP 12 (2018) 024 [arXiv: 1810.03626] [INSPIRE].

[34] B. Dutta et al., Dark matter signals from timing spectra at neutrino experiments, arXiv: 1906.10745 [INSPIRE].

[35] L.M. Sehgal, Differences in the coherent interactions of $\nu_{e}, \nu_{\mu}$ and $\nu_{\tau}$, Phys. Lett. B 162 (1985) 370 .

[36] R. Garoby et al., The European Spallation Source design, Phys. Scripta 93 (2018) 014001 [INSPIRE].

[37] R.L. Burman and P. Plischke, Neutrino fluxes from a high-intensity spallation neutron facility, Nucl. Instrum. Meth. A 398 (1997) 147 [INSPIRE].

[38] R.L. Burman and P. Plischke, Neutrino flux calculations for the proposed European Spallation Source, FZKA-5834 (1996).

[39] R.E. Prael and H. Lichtenstein, The LAHET Code System, LA-UR-89-30 (1989).

[40] R.L. Burman and W.C. Louis, Neutrino physics at meson factories and spallation neutron sources, J. Phys. G 29 (2003) 2499 [INSPIRE]. 
[41] D. Pelowitz et al., MCNPX user's manual version 2.7.0, LA-CP-11-00438 (2011).

[42] GEANT4 collaboration, GEANT4: a simulation toolkit, Nucl. Instrum. Meth. A 506 (2003) 250 [INSPIRE].

[43] T.T. Böhlen et al., The FLUKA code: developments and challenges for high energy and medical applications, Nucl. Data Sheets 120 (2014) 211 [inSPIRE].

[44] K. Batkov, A. Takibayev, L. Zanini and F. Mezei, Unperturbed moderator brightness in pulsed neutron sources, Nucl. Instrum. Meth. A 729 (2013) 500.

[45] D.D. DiJulio et al., Benchmarking GEANT4 for spallation neutron source calculations, J. Phys. Conf. Ser. 746 (2016) 012032.

[46] HARP collaboration, Large-angle production of charged pions with incident pion beams on nuclear targets, Phys. Rev. C 80 (2009) 065207 [arXiv: 0907.1428] [INSPIRE].

[47] A. Bolshakova et al., Cross-sections of large-angle hadron production in proton- and pion-nucleus interactions VIII: aluminium nuclei and beam momenta from $\pm 3 \mathrm{GeV} / \mathrm{c}$ to $\pm 15 \mathrm{GeV} / \mathrm{c}$, Eur. Phys. J. C 72 (2012) 1882 [arXiv:1110.6753] [InSPIRE].

[48] HARP-CDP collaboration, HARP-CDP hadroproduction data: Comparison with FLUKA and GEANT4 simulations, Eur. Phys. J. C 70 (2010) 543 [arXiv:1006.3429] [INSPIRE].

[49] J.C. David, Spallation reactions: a successful interplay between modeling and applications, Eur. Phys. J. A 51 (2015) 68 [arXiv:1505.03282] [INSPIRE].

[50] D. Mancusi et al., On the role of secondary pions in spallation targets, Eur. Phys. J. A 53 (2017) 80 [arXiv: 1603.05453] [INSPIRE].

[51] HARP-CDP Group collaboration, Revisiting the 'LSND anomaly' I: impact of new data, Phys. Rev. D 85 (2012) 092008 [arXiv:1110.4265] [InSPIRE].

[52] J. Newby, A precision neutrino laboratory at the Spallation Neutron Source, talk given at The Magnificent CEעNS workshop, November 2-3, University of Chicago, Chicago U.S.A. (2018).

[53] Y. Efremenko, SNS neutrino fluxes, talk given at NuEclipse Workshop, August 20-22, University of Tennessee, Knoxville, U.S.A. (2017).

[54] COHERENT collaboration, COHERENT plans for $\mathrm{D}_{2} \mathrm{O}$ at the Spallation neutron source, talk given at Meeting of the Division of Particles and Fields of the American Physical Society (DPF2019), July 29-August 2, Boston, Massachusetts, U.S.A (2019), arXiv: 1910.00630 [INSPIRE].

[55] K. Scholberg, Coherent neutrino scattering, talk given at Topics in Astroparticle and Underground Physics (TAUP 2019), September 8-14, Toyama, Japan (2019).

[56] M.R. Heath, A first search for coherent elastic neutrino-nucleus scattering with liquid argon, Ph.D. thesis, Indiana University, Bloomington, U.S.A. (2019).

[57] COHERENT collaboration, First constraint on coherent elastic neutrino-nucleus scattering in argon, Phys. Rev. D 100 (2019) 115020 [arXiv: 1909.05913] [INSPIRE].

[58] C. Amsler et al., Temperature dependence of pure CsI: scintillation light yield and decay time, Nucl. Instrum. Meth. A 480 (2002) 494 [inSPIRE].

[59] M. Moszynski et al., Energy resolution and non-proportionality of the light yield of pure csi at liquid nitrogen temperatures, Nucl. Instrum. Meth. A 537 (2005) 357. 
[60] M. Moszynski et al., Application of large area avalanche photodiodes to study scintillators at liquid nitrogen temperatures, Nucl. Instrum. Meth. A 504 (2003) 307.

[61] P. Nadeau, Cryogenic scintillators for rare-event searches, Ph.D. thesis, Queen's University, Ontario, Canada (2015).

[62] M. Clar et al., Particle detection at cryogenic temperatures with undoped CsI, Nucl. Instrum. Meth. A 901 (2018) 6 [arXiv:1709.04020] [INSPIRE].

[63] J. Liu, M. Yamashita and A.K. Soma, Light yield of an undoped CsI crystal coupled directly to a photomultiplier tube at 77 Kelvin, 2016 JINST 11 P10003 [arXiv:1608.06278] [INSPIRE].

[64] C.L. Woody et al., Readout techniques and radiation damage of undoped cesium iodide, IEEE Trans. Nucl. Sci. 37 (1990) 492 [INSPIRE].

[65] X. Zhang et al., Tracking with the fastest light in the JUNO central detector, Radiat. Detect. Technol. Meth. 2 (2018) 13

[66] V.B. Mikhailik et al., Luminescence and scintillation properties of CsI - a potential cryogenic scintillator, Phys. Status Solidi B 252 (2015) 804 [arXiv:1411.6246] [inSPIRE].

[67] S.S. Gridin et al., Channels of energy losses and relaxation in CSi:a scintillators ( $a=T l$, In), IEEE Trans. Nucl. Sci. 61 (2014) 246.

[68] P. Dorenbos, Light output and energy resolution of $\mathrm{Ce}^{3+}$-doped scintillators, Nucl. Instrum. Meth. A 486 (2002) 208.

[69] G. Angloher et al., A CsI low temperature detector for dark matter search, Astropart. Phys. 84 (2016) 70 [arXiv:1602.08884] [INSPIRE].

[70] C.K. Ong, K.S. Song, R. Monnier and A.M. Stoneham, Electronic structure and luminescence of CsI:Na, J. Phys. C 12 (1979) 4641.

[71] J.I. Collar, A.R.L. Kavner and C.M. Lewis, Response of CsI[Na] to nuclear recoils: impact on coherent elastic neutrino-nucleus scattering (CEvNS), Phys. Rev. D 100 (2019) 033003 [arXiv: 1907.04828] [INSPIRE].

[72] Radiation Monitoring Devices, RMD Inc. Watertown, U.S.A. .

[73] Y. Jin et al., Study of a pure CsI crystal readout by APD for Belle II end cap ECL upgrade, Nucl. Instrum. Meth. A 824 (2016) 691 [INSPIRE].

[74] Y. Jin Study of a scintillation counter consisting of a pure CsI crystal and avalanche photodiodes, Master's thesis, University of Tokyo, Tokyo, Japan (2015).

[75] N. Osakabe et al., Saturation of multiplication mechanism in silicon avalanche photodiodes used for single electron detection, Rev. Sci. Instrum. 69 (1998) 2898.

[76] L. Yang et al., Performance of a large-area avalanche photodiode at low temperature for scintillation detection, Nucl. Instrum. Meth. A 508 (2003) 388 [INSPIRE].

[77] A. Wright, The photomultiplier handbook, Oxford University Press, Oxford U.K. (2017).

[78] https://www.luminnotech.com/products.

[79] R. Neilson et al., Characterization of large area APDs for the EXO-200 detector, Nucl. Instrum. Meth. A 608 (2009) 68 [arXiv:0906.2499] [INSPIRE].

[80] Specification sheet for R8520-406/R8520-506 PMTs, https://www.hamamatsu.com. 
[81] M. Ibe, W. Nakano, Y. Shoji and K. Suzuki, Migdal Effect in Dark Matter Direct Detection Experiments, JHEP 03 (2018) 194 [arXiv:1707.07258] [INSPIRE].

[82] S.A. Ponomarenko et al., Nanostructured organosilicon luminophores and their application in highly efficient plastic scintillators, Nature Sci. Rep. 4 (2014) 6549.

[83] T.Y. Starikova et al., A novel highly efficient nanostructured organosilicon luminophore with unusually fast photoluminescence, J. Mater. Chem. C 4 (2016) 4699.

[84] S. A. Ponomarenko et al., Nanostructured organosilicon luminophores for efficient and fast elementary particles photodetectors, Proc. SPIE 10344 (2017) 49.

[85] S.A. Ponomarenko et al., Nanostructured organosilicon luminophores as a new concept of nanomaterials for highly efficient down-conversion of light, Proc. SPIE 9545 (2015) 8.

[86] O. Borshchev, N. Surin, M. Skorotetcky and S. Ponomarenko., High-efficient optical wavelength shifters: design, properties, application, INEOS OPEN 2 (2019) 112.

[87] M. McClish et al., Characterization of very large silicon avalanche photodiodes, IEEE Symp. Conf. Rec. Nucl. Sci. 2 (2004) 1270.

[88] Y. Yanagida and H. Yoshimoto, Reusing KTeV CsI crystals for J-PARC KOTO experiment, PoS (KAON09) 021.

[89] DAMIC collaboration, Search for low-mass WIMPs in a $0.6 \mathrm{~kg}$ day exposure of the DAMIC experiment at SNOLAB, Phys. Rev. D 94 (2016) 082006 [arXiv:1607.07410] [InSPIRE].

[90] DAMIC collaboration, Constraints on light dark matter particles interacting with electrons from DAMIC at SNOLAB, Phys. Rev. Lett. 123 (2019) 181802 [arXiv:1907.12628] [INSPIRE].

[91] SENSEI collaboration, SENSEI: direct-detection constraints on sub-GeV dark matter from a shallow underground run using a prototype skipper-CCD, Phys. Rev. Lett. 122 (2019) 161801 [arXiv: 1901.10478] [INSPIRE].

[92] CONNIE collaboration, Exploring low-energy neutrino physics with the coherent neutrino nucleus interaction experiment, Phys. Rev. D 100 (2019) 092005 [arXiv:1906.02200] [INSPIRE].

[93] https://damic.uchicago.edu/index.php

[94] P. Privitera, The DAMIC-M dark matter experiment, talk given at Topics in Astroparticle and Underground Physics (TAUP 2019), September 8-14, Toyama, Japan (2019).

[95] R.D. Ryan, Precision measurements of the ionization energy and its temperature variation in high purity silicon radiation detectors, IEEE Trans. Nucl. Sci. 20 (1973) 473.

[96] SENSEI collaboration, Single-electron and single-photon sensitivity with a silicon Skipper CCD, Phys. Rev. Lett. 119 (2017) 131802 [arXiv:1706.00028] [INSPIRE].

[97] A.E. Chavarria et al., Measurement of the ionization produced by sub-keV silicon nuclear recoils in a CCD dark matter detector, Phys. Rev. D 94 (2016) 082007 [arXiv: 1608.00957] [INSPIRE].

[98] J.I. Collar, Applications of an ${ }^{88} Y / B e$ photo-neutron calibration source to dark matter and neutrino experiments, Phys. Rev. Lett. 110 (2013) 211101 [arXiv:1303.2686] [INSPIRE].

[99] C.W. Leitz et al., Development of germanium charge-coupled devices, Proc. SPIE 10709 (2018) 1070908. 
[100] J.J. Gomez-Cadenas, Status and prospects of the NEXT experiment for neutrinoless double beta decay searches, 2019, arXiv: 1906.01743 [INSPIRE].

[101] P. Sorensen, Electron train backgrounds in liquid xenon dark matter search detectors are indeed due to thermalization and trapping, arXiv:1702.04805 [INSPIRE].

[102] J. Xu et al., Electron extraction efficiency study for dual-phase xenon dark matter experiments, Phys. Rev. D 99 (2019) 103024 [arXiv:1904.02885] [InSPIRE].

[103] RED-100 collaboration, First ground-level laboratory test of the two-phase xenon emission detector RED-100, arXiv: 1910.06190 [INSPIRE].

[104] NEXT collaboration, Ionization and scintillation of nuclear recoils in gaseous xenon, Nucl. Instrum. Meth. A 793 (2015) 62 [arXiv:1409.2853] [INSPIRE].

[105] NEXT collaboration, Calibration of the NEXT-White detector using ${ }^{83 m} \mathrm{Kr}$ decays, 2018 JINST 13 P10014 [arXiv: 1804.01780] [INSPIRE].

[106] J.J. Gomez-Cadenas, F. Monrabal Capilla and P. Ferrario, High pressure gas xenon TPCs for double beta decay searches, Front. in Phys. 7 (2019) 51 [arXiv:1903.02435] [INSPIRE].

[107] NEXT collaboration, The Next White (NEW) detector, 2018 JINST 13 P12010 [arXiv: 1804. 02409] [INSPIRE].

[108] P.S. Barbeau, J.I. Collar and O. Tench, Large-mass ultra-low noise germanium detectors: performance and applications in neutrino and astroparticle physics, JCAP 09 (2007) 009 [nucl-ex/0701012] [INSPIRE].

[109] MaJorana collaboration, A search for neutrinoless double-beta decay in ${ }^{76} \mathrm{Ge}$ with $26 \mathrm{~kg}$-yr of exposure from the Majorana DEMONSTRATOR, Phys. Rev. C 100 (2019) 025501 [arXiv: 1902.02299] [INSPIRE].

[110] GERDA collaboration, Improved limit on neutrinoless double- $\beta$ decay of ${ }^{76} \mathrm{Ge}$ from GERDA Phase II, Phys. Rev. Lett. 120 (2018) 132503 [arXiv: 1803.11100] [INSPIRE].

[111] H. T.-K. Wong, Taiwan EXperiment On NeutrinO - History and prospects, The Universe 3 (2015) 22 [arXiv:1608.00306] [INSPIRE].

[112] J. Hakenmüller et al., Neutron-induced background in the CONUS experiment, Eur. Phys. J. C 79 (2019) 699 [arXiv: 1903.09269] [INSPIRE].

[113] CoGENT collaboration, CoGeNT: a search for low-mass dark matter using p-type point contact germanium detectors, Phys. Rev. D 88 (2013) 012002 [arXiv:1208.5737] [inSPIRE].

[114] CDEX collaboration, Limits on light weakly interacting massive particles from the first $102.8 \mathrm{~kg} \times$ day data of the CDEX-10 experiment, Phys. Rev. Lett. 120 (2018) 241301 [arXiv: 1802.09016] [INSPIRE].

[115] G. Ilie, Mirion Ge detectors, talk given at the $3^{\text {rd }}$ PIRE-GEMADARC workshop, 5 December, Knoxville, U.S.A. (2018),

[116] R. Cooper, D. Radford, P. Hausladen and K. Lagergren, A novel hpge detector for gamma-ray tracking and imaging, Nucl. Instrum. Meth. A 665 (2011) 25.

[117] C. Awe et al., Liquid scintillator response to proton recoils in the 10-100 keV range, Phys. Rev. C 98 (2018) 045802 [arXiv: 1804.06457] [INSPIRE].

[118] B.J. Scholz et al., Measurement of the low-energy quenching factor in germanium using an ${ }^{88}$ Y/Be photoneutron source, Phys. Rev. D 94 (2016) 122003 [arXiv:1608.03588] [INSPIRE]. 
[119] W.J. Bolte et al., Development of bubble chambers with enhanced stability and sensitivity to low-energy nuclear recoils, Nucl. Instrum. Meth. A 577 (2007) 569 [astro-ph/0503398] [INSPIRE].

[120] COUPP collaboration, Improved spin-dependent WIMP limits from a bubble chamber, Science 319 (2008) 933 [arXiv: 0804.2886] [INSPIRE].

[121] PICO collaboration, Dark matter search results from the complete exposure of the PICO-60 $C_{3} F_{8}$ bubble chamber, Phys. Rev. D 100 (2019) 022001 [arXiv:1902.04031] [INSPIRE].

[122] PICO collaboration, Data-driven modeling of electron recoil nucleation in PICO $C_{3} F_{8}$ bubble chambers, Phys. Rev. D 100 (2019) 082006 [arXiv: 1905.12522] [INSPIRE].

[123] D. Baxter et al., First demonstration of a scintillating xenon bubble chamber for detecting dark matter and coherent elastic neutrino-nucleus scattering, Phys. Rev. Lett. 118 (2017) 231301 [arXiv: 1702.08861] [INSPIRE].

[124] E. Dahl, Progress on liquid-noble bubble chambers for $C E \nu N S$, talk given at The magnificent

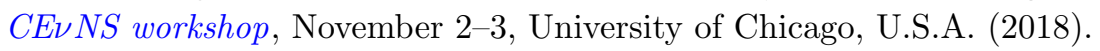

[125] COUPP collaboration, Direct measurement of the bubble nucleation energy threshold in a $\mathrm{CF}_{3} I$ bubble chamber, Phys. Rev. D 88 (2013) 021101 [arXiv: 1304.6001] [INSPIRE].

[126] P. Coloma et al., Curtailing the dark side in non-standard neutrino interactions, JHEP 04 (2017) 116 [arXiv: 1701.04828] [INSPIRE].

[127] A.N. Khan and W. Rodejohann, New physics from COHERENT data with an improved quenching factor, Phys. Rev. D 100 (2019) 113003 [arXiv: 1907.12444] [INSPIRE].

[128] Particle Data Group collaboration, Review of particle physics, Phys. Rev. D 98 (2018) 030001 [INSPIRE].

[129] J. Erler and R. Ferro-Hernández, Weak mixing angle in the Thomson limit, JHEP 03 (2018) 196 [arXiv:1712.09146] [INSPIRE].

[130] C.J. Horowitz, K.J. Coakley and D.N. McKinsey, Supernova observation via neutrino-nucleus elastic scattering in the CLEAN detector, Phys. Rev. D 68 (2003) 023005 [astro-ph/0302071] [INSPIRE].

[131] R.H. Helm, Inelastic and elastic scattering of 187 Mev electrons from selected even-even nuclei, Phys. Rev. 104 (1956) 1466 [inSPIRE].

[132] F. Izraelevitch et al., A measurement of the ionization efficiency of nuclear recoils in silicon, 2017 JINST 12 P06014 [arXiv:1702.00873] [INSPIRE].

[133] S. Weinberg, Baryon and lepton nonconserving processes, Phys. Rev. Lett. 43 (1979) 1566 [INSPIRE].

[134] P. Coloma and T. Schwetz, Generalized mass ordering degeneracy in neutrino oscillation experiments, Phys. Rev. D 94 (2016) 055005 [Erratum ibid. D 95 (2017) 079903] [arXiv: 1604.05772] [INSPIRE].

[135] M.C. Gonzalez-Garcia and M. Maltoni, Determination of matter potential from global analysis of neutrino oscillation data, JHEP 09 (2013) 152 [arXiv:1307.3092] [INSPIRE].

[136] P. Bakhti and Y. Farzan, Shedding light on LMA-dark solar neutrino solution by medium baseline reactor experiments: JUNO and RENO-50, JHEP 07 (2014) 064 [arXiv: 1403.0744] [INSPIRE]. 
[137] J. Barranco, O.G. Miranda and T.I. Rashba, Probing new physics with coherent neutrino scattering off nuclei, JHEP 12 (2005) 021 [hep-ph/0508299] [INSPIRE].

[138] NuTEV collaboration, A precise determination of electroweak parameters in neutrino nucleon scattering, Phys. Rev. Lett. 88 (2002) 091802 [Erratum ibid. 90 (2003) 239902] [hep-ex/0110059] [INSPIRE].

[139] CHARM collaboration, Experimental verification of the universality of $\nu_{e}$ and $\nu_{\mu}$ coupling to the neutral weak current, Phys. Lett. B 180 (1986) 303 [INSPIRE].

[140] Y. Farzan, A model for large non-standard interactions of neutrinos leading to the LMA-dark solution, Phys. Lett. B 748 (2015) 311 [arXiv:1505. 06906] [INSPIRE].

[141] Y. Farzan and I.M. Shoemaker, Lepton flavor violating non-standard interactions via light mediators, JHEP 07 (2016) 033 [arXiv: 1512.09147] [INSPIRE].

[142] COHERENT collaboration, COHERENT collaboration data release from the first observation of coherent elastic neutrino-nucleus scattering, arXiv:1804.09459 [INSPIRE].

[143] C.S. Wood et al., Measurement of parity nonconservation and an anapole moment in cesium, Science $\mathbf{2 7 5}$ (1997) 1759 [INSPIRE].

[144] V.A. Dzuba, J.C. Berengut, V.V. Flambaum and B. Roberts, Revisiting parity non-conservation in cesium, Phys. Rev. Lett. 109 (2012) 203003 [arXiv:1207.5864] [INSPIRE].

[145] SLAC E158 collaboration, Precision measurement of the weak mixing angle in Moller scattering, Phys. Rev. Lett. 95 (2005) 081601 [hep-ex/0504049] [INSPIRE].

[146] PVDIS collaboration, Measurement of parity violation in electron-quark scattering, Nature 506 (2014) 67.

[147] C. Giunti and A. Studenikin, Neutrino electromagnetic interactions: a window to new physics, Rev. Mod. Phys. 87 (2015) 531 [arXiv: 1403.6344] [INSPIRE].

[148] G. Degrassi, A. Sirlin and W.J. Marciano, Effective electromagnetic form-factor of the neutrino, Phys. Rev. D 39 (1989) 287 [inSPIRE].

[149] P. Vogel and J. Engel, Neutrino electromagnetic form-factors, Phys. Rev. D 39 (1989) 3378 [INSPIRE].

[150] K.A. Kouzakov and A.I. Studenikin, Electromagnetic properties of massive neutrinos in low-energy elastic neutrino-electron scattering, Phys. Rev. D 95 (2017) 055013 [Erratum ibid. D 96 (2017) 099904] [arXiv: 1703.00401] [INSPIRE].

[151] J. Bernabeu, L.G. Cabral-Rosetti, J. Papavassiliou and J. Vidal, On the charge radius of the neutrino, Phys. Rev. D 62 (2000) 113012 [hep-ph/0008114] [INSPIRE].

[152] J. Bernabeu, J. Papavassiliou and J. Vidal, On the observability of the neutrino charge radius, Phys. Rev. Lett. 89 (2002) 101802 [Erratum ibid. 89 (2002) 229902] [hep-ph/0206015] [INSPIRE].

[153] J. Bernabeu, J. Papavassiliou and J. Vidal, The neutrino charge radius is a physical observable, Nucl. Phys. B 680 (2004) 450 [hep-ph/0210055] [INSPIRE].

[154] A.G. Beda et al., The results of search for the neutrino magnetic moment in GEMMA experiment, Adv. High Energy Phys. 2012 (2012) 350150. 